\title{
Equilibrium stacks for a three-person game on a product of staircase-function continuous and finite strategy spaces
}

\author{
Vadim Romanuke *
}

\begin{abstract}
A method of solving a three-person game defined on a product of staircase-function strategy spaces is presented. The spaces can be finite and continuous. The method is based on stacking equilibria of "short" three-person games, each defined on an interval where the pure strategy value is constant. In the case of finite three-person games, which factually are trimatrix games, the equilibria are considered in general terms, so they can be in mixed strategies as well. The stack is any interval-wise combination (succession) of the respective equilibria of the "short" trimatrix games. Apart from the stack, there are no other equilibria in this "long" trimatrix game. An example is presented to show how the stacking is fulfilled for a case of when every "short" trimatrix game has a pure-strategy equilibrium. The presented method, further "breaking" the initial "long" game defined on a product of staircase-function finite spaces, is far more tractable than a straightforward approach to solving directly the "long" trimatrix game would be.
\end{abstract}

Keywords: game theory, payoff functional, staircase-function strategy, three-person game, trimatrix game.

\section{Introduction}

In rationalizing the distribution of limited resources, three-person noncooperative games are applied as often as a game model representing two sides becomes impossible [15, 22]. Trimatrix games are the simplest three-person games wherein equilibrium, efficiency, profitability, and eventual optimality of their solutions are well-studied [15, 22, 26]. An infinite or continuous three-person game (where the players' payoff functions are hypersurfaces, which may have also discontinuities, defined on finite-dimensional Euclidean subspaces) is far more complicated as, opposed to a trimatrix game, an equilibrium is not always determinable and feasible $[23,10]$. Therefore, the best choice is to approximate such a game to a finite one, which always has an equilibrium [22, 23].

A finite three-person noncooperative game is easily rendered to a trimatrix game [23, 12]. There are a few approaches to exactly solve a trimatrix game $[22,10,12]$. However, a trimatrix game equilibrium, if it is in mixed strategies, is not always practicable due to

* Polish Naval Academy, Gdynia, Poland, v.romanuke@amw.gdynia.pl 
finite horizon of the game iterations (rounds, plays, actions, etc.) [14, 18, 15, 13]. Besides, there is a difficulty of practicing the mixed-strategy equilibrium wherein the pure strategy is a complex action through time rather than an elementary action $[25,18]$. Moreover, if the game has more than one equilibrium, a problem of the equilibrium selection arises $[18,12$, 4]. Furthermore, if at least two equilibria are symmetric, they may be quite unstable due to cooperation between the players is excluded $[22,23,15,12]$.

The trimatrix game rendering can be fulfilled regardless of the pure strategy complexity $[18,15,12,21]$. Obviously, such rendering is impossible if the set of the player's strategies is either infinite or continuous. If the player's pure strategy is a function (commonly, it is a function of time), and every player possesses a finite set of such function-strategies, the rendering results in huge three-dimensional payoff matrices. This is a far more complicated three-person game. In this game, the player's payoff is a functional $[18,25,19]$. Each player's functional maps every triplet of functions (pure strategies of the players defined on a time interval) into a real value. The same is done if the pure strategy sets are infinite or continuous.

A finite game is not obtained by just breaking a time interval, on which the pure strategy is defined, into a set of subintervals, on which the strategy could be approximately considered constant. This is so because of the continuity of possible values of the strategy on a subinterval. However, the continuity might be removed also by sampling $[21,16]$. Then the set of function-strategies becomes finite. This results in a finite three-person noncooperative game. It is clear that the size and properties of this game strongly depend on both samplings along time axis and strategy value axis $[19,16]$.

Regardless of the form of pure strategies used in the game, the number of factual actions of the players in any game has a natural limit $[15,22,23,14,18]$. Nevertheless, if the rules of a system which is game-modeled are defined and administered beforehand, the administrator is likely to define (or constrain) the form of the strategies players will use [16, $24,25]$.

\section{Motivation}

In the simplest case, the player's pure strategy is an elementary action whose duration is negligibly short and thus is represented as just a time point. This case is exhaustively studied as bimatrix, trimatrix, and dyadic games $[23,22,18,15,20]$. In a more complicated case, the player's pure strategy is a function of time $[18,16,19]$, so the player's action is a complex process whose duration cannot be reduced to a time point. A way to appropriately administer the players' actions is to constrain them to staircase functions whose points of discontinuities (breakpoints) have to be the same for all the players [19, 20, 27, 28]. Along with the discrete time, possible values of the player's pure strategy should be discrete as well. Then the game can be represented as a trimatrix game, in which the player's selection of a pure strategy means using a staircase function on a time interval whereon every pure strategy is defined.

Obviously, the number of the player's pure strategies in the trimatrix staircase-function game grows immensely as the number of breakpoints ("stair" intervals) or/and the number of possible values of the player's pure strategy increases. For instance, if the number of intervals is 5 , and the number of possible values of the player's pure strategy is just 4 , then 
there are $4^{5}=1024$ possible pure strategies at this player, where every strategy is a 5 -interval 4-staircased function of time. The respective trimatrix

$$
1024 \times 1024 \times 1024 \text { game }
$$

even in this trivialized case appears to be big enough. Meanwhile, it may take between 2.7 to 3.9 seconds to solve a $25 \times 25 \times 25$ game on a laptop with an Intel Core i7 processor. When every strategy, say, is a 6-interval 8-staircased function of time, the respective trimatrix

\section{$262144 \times 262144 \times 262144$ staircase-function game}

appears to be intractably gigantic even in this example (which is a more real but nonetheless is still not a high-probability process). There are

$$
18014398509481984
$$

(more than 18 quadrillion) situations in the game. This is a simple example of the intractability. This means that, instead of rendering a three-person game defined on a product of staircase-function finite spaces to a trimatrix game, a tractable method of solving it should be suggested.

\section{Objective and tasks to be fulfilled}

Issuing from the impracticability of rendering finite three-person games with staircasefunction strategies to trimatrix games, the objective is to develop a tractable method of solving three-person games defined on a product of staircase-function finite spaces. For achieving the objective, the following six tasks are to be fulfilled:

1. To formalize a three-person game, in which the players' strategies are staircase functions. In such a game, the set of the player's pure strategies is a continuum of staircase functions of time. Herein, the time can be thought of as it is discrete.

2. To discretize the set of possible values of the player's pure strategy so that the game be defined on a product of staircase-function finite spaces.

3. To formalize a method of solving three-person games defined on a product of staircase-function finite spaces.

4. To exemplify it by showing how the method is used to solve a finite three-person game with staircase-function strategies.

5. To discuss applicability and significance of the method, as well as its possible drawbacks and limitations.

6. To make an appropriate conclusion on it.

\section{A three-person game with staircase-function strategies}

In a three-person game, in which the player's pure strategy is a function of time, let each of the players use time-varying strategies defined almost everywhere on interval $\left[t_{1} ; t_{2}\right]$ by 
$t_{2}>t_{1}$. Denote a strategy of the first, second, and third players by $x(t), y(t)$, and $z(t)$, respectively. These functions are presumed to be bounded, i. e.

$$
\begin{gathered}
a_{\min } \leqslant x(t) \leqslant a_{\max } \text { by } a_{\min }<a_{\max }, \\
b_{\min } \leqslant y(t) \leqslant b_{\max } \text { by } b_{\min }<b_{\max },
\end{gathered}
$$

and

$$
c_{\min } \leqslant z(t) \leqslant c_{\max } \text { by } c_{\min }<c_{\max },
$$

defined almost everywhere on $\left[t_{1} ; t_{2}\right]$. Besides, the square of the function-strategy is presumed to be Lebesgue-integrable. Thus, pure strategies of the player belong to a rectangular functional space of functions of time:

$$
\begin{aligned}
& X=\left\{x(t), t \in\left[t_{1} ; t_{2}\right], t_{1}<t_{2}: a_{\text {min }} \leqslant x(t) \leqslant a_{\text {max }} \text { by } a_{\text {min }}<a_{\text {max }}\right\} \subset \mathbb{L}_{2}\left[t_{1} ; t_{2}\right], \\
& Y=\left\{y(t), t \in\left[t_{1} ; t_{2}\right], t_{1}<t_{2}: b_{\text {min }} \leqslant y(t) \leqslant b_{\text {max }} \text { by } b_{\text {min }}<b_{\text {max }}\right\} \subset \mathbb{L}_{2}\left[t_{1} ; t_{2}\right],
\end{aligned}
$$

and

$$
Z=\left\{z(t), t \in\left[t_{1} ; t_{2}\right], t_{1}<t_{2}: c_{\min } \leqslant z(t) \leqslant c_{\max } \text { by } c_{\min }<c_{\max }\right\} \subset \mathbb{L}_{2}\left[t_{1} ; t_{2}\right]
$$

are the sets of the players' pure strategies, respectively.

The player's payoff in situation

$$
\{x(t), y(t), z(t)\}
$$

is presumed to be an integral functional $[19,18,16,22,2]$. Thus, the first, second, and third players' payoffs are

$$
\begin{aligned}
& F(x(t), y(t), z(t))=\int_{\left[t_{1} ; t_{2}\right]} f(x(t), y(t), z(t), t) d \mu(t), \\
& G(x(t), y(t), z(t))=\int_{\left[t_{1} ; t_{2}\right]} g(x(t), y(t), z(t), t) d \mu(t), \\
& H(x(t), y(t), z(t))=\int_{\left[t_{1} ; t_{2}\right]} h(x(t), y(t), z(t), t) d \mu(t),
\end{aligned}
$$

respectively, where

$$
\begin{gathered}
f(x(t), y(t), z(t), t), \\
g(x(t), y(t), z(t), t), \\
h(x(t), y(t), z(t), t)
\end{gathered}
$$


are functions of $x(t), y(t), z(t)$, explicitly including time $t$. Therefore, the continuous three-person game

$$
\langle\{X, Y, Z\},\{F(x(t), y(t), z(t)), G(x(t), y(t), z(t)), H(x(t), y(t), z(t))\}\rangle
$$

is defined on product

$$
X \times Y \times Z \subset \mathbb{L}_{2}\left[t_{1} ; t_{2}\right] \times \mathbb{L}_{2}\left[t_{1} ; t_{2}\right] \times \mathbb{L}_{2}\left[t_{1} ; t_{2}\right]
$$

of rectangular functional spaces (4) - (6) of players' pure strategies.

First, it is presumed that game (14) is administered so that the players are forced to use pure strategies $x(t)$ and $y(t)$ and $z(t)$ such that they all change their values for a finite number of times. Denote by $N$ the number of intervals at which the player's pure strategy is constant, where $N \in \mathbb{N} \backslash\{1\}$. Then the player's pure strategy is a staircase function having only $N$ different values. If $\left\{\tau^{(i)}\right\}_{i=1}^{N-1}$ are time points at which the staircase-function strategy changes its value, where

$$
t_{1}=\tau^{(0)}<\tau^{(1)}<\tau^{(2)}<\ldots<\tau^{(N-1)}<\tau^{(N)}=t_{2},
$$

then

$$
\left\{x\left(\tau^{(i)}\right)\right\}_{i=0}^{N},\left\{y\left(\tau^{(i)}\right)\right\}_{i=0}^{N},\left\{z\left(\tau^{(i)}\right)\right\}_{i=0}^{N}
$$

are the values of the players' strategies in a play-off of game (14). The staircase-function strategies are right-continuous $[2,17,16]$ :

$$
\begin{aligned}
& \lim _{\substack{\varepsilon>0 \\
\varepsilon \rightarrow 0}} x\left(\tau^{(i)}+\varepsilon\right)=x\left(\tau^{(i)}\right), \\
& \lim _{\substack{\varepsilon>0 \\
\varepsilon \rightarrow 0}} y\left(\tau^{(i)}+\varepsilon\right)=y\left(\tau^{(i)}\right), \\
& \lim _{\substack{\varepsilon>0 \\
\varepsilon \rightarrow 0}} z\left(\tau^{(i)}+\varepsilon\right)=z\left(\tau^{(i)}\right),
\end{aligned}
$$

for $i=\overline{1, N-1}$, whereas

$$
\begin{aligned}
& \lim _{\substack{\varepsilon>0 \\
\varepsilon \rightarrow 0}} x\left(\tau^{(i)}-\varepsilon\right) \neq x\left(\tau^{(i)}\right), \\
& \lim _{\substack{\varepsilon>0 \\
\varepsilon \rightarrow 0}} y\left(\tau^{(i)}-\varepsilon\right) \neq y\left(\tau^{(i)}\right), \\
& \lim _{\substack{\varepsilon>0 \\
\varepsilon \rightarrow 0}} z\left(\tau^{(i)}-\varepsilon\right) \neq z\left(\tau^{(i)}\right),
\end{aligned}
$$


for $i=\overline{1, N-1}$. As an exception,

$$
\begin{aligned}
& \lim _{\substack{\varepsilon>0 \\
\varepsilon \rightarrow 0}} x\left(\tau^{(N)}-\varepsilon\right)=x\left(\tau^{(N)}\right), \\
& \lim _{\substack{\varepsilon>0 \\
\varepsilon \rightarrow 0}} y\left(\tau^{(N)}-\varepsilon\right)=y\left(\tau^{(N)}\right), \\
& \lim _{\substack{\varepsilon>0 \\
\varepsilon \rightarrow 0}} z\left(\tau^{(N)}-\varepsilon\right)=z\left(\tau^{(N)}\right),
\end{aligned}
$$

so

$$
\begin{aligned}
& x\left(\tau^{(N-1)}\right)=x\left(\tau^{(N)}\right), \\
& y\left(\tau^{(N-1)}\right)=y\left(\tau^{(N)}\right), \\
& z\left(\tau^{(N-1)}\right)=z\left(\tau^{(N)}\right) .
\end{aligned}
$$

Then constant values (17) by (16) mean that game (14) can be thought of as it is a succession of $N$ continuous games

$$
\left\langle\left\{\left[a_{\min } ; a_{\max }\right],\left[b_{\min } ; b_{\max }\right],\left[c_{\min } ; c_{\max }\right]\right\},\left\{F\left(\alpha_{i}, \beta_{i}, \gamma_{i}\right), G\left(\alpha_{i}, \beta_{i}, \gamma_{i}\right), H\left(\alpha_{i}, \beta_{i}, \gamma_{i}\right)\right\}\right\rangle
$$

defined on parallelepiped

$$
\left[a_{\min } ; a_{\max }\right] \times\left[b_{\min } ; b_{\max }\right] \times\left[c_{\min } ; c_{\max }\right]
$$

by

$$
\begin{gathered}
\alpha_{i}=x(t) \in\left[a_{\min } ; a_{\max }\right] \text { and } \beta_{i}=y(t) \in\left[b_{\min } ; b_{\max }\right] \text { and } \gamma_{i}=z(t) \in\left[c_{\min } ; c_{\max }\right] \\
\forall t \in\left[\tau^{(i-1)} ; \tau^{(i)}\right) \text { for } i=\overline{1, N-1} \text { and } \forall t \in\left[\tau^{(N-1)} ; \tau^{(N)}\right],
\end{gathered}
$$

where the factual players' payoffs in situation $\left\{\alpha_{i}, \beta_{i}, \gamma_{i}\right\}$ are

$$
F\left(\alpha_{i}, \beta_{i}, \gamma_{i}\right)=\int_{\left[\tau^{(i-1)} ; \tau^{(i)}\right)} f\left(\alpha_{i}, \beta_{i}, \gamma_{i}, t\right) d \mu(t) \quad \forall i=\overline{1, N-1}
$$

by

$$
\begin{gathered}
F\left(\alpha_{N}, \beta_{N}, \gamma_{N}\right)=\int_{\left[\tau^{(N-1)} ; \tau^{(N)}\right]} f\left(\alpha_{N}, \beta_{N}, \gamma_{N}, t\right) d \mu(t), \\
G\left(\alpha_{i}, \beta_{i}, \gamma_{i}\right)=\int_{\left[\tau^{(i-1)} ; \tau^{(i)}\right)} g\left(\alpha_{i}, \beta_{i}, \gamma_{i}, t\right) d \mu(t) \quad \forall i=\overline{1, N-1}
\end{gathered}
$$

by 


$$
G\left(\alpha_{N}, \beta_{N}, \gamma_{N}\right)=\int_{\left[\tau^{(N-1)} ; \tau^{(N)}\right]} g\left(\alpha_{N}, \beta_{N}, \gamma_{N}, t\right) d \mu(t)
$$

and

$$
H\left(\alpha_{i}, \beta_{i}, \gamma_{i}\right)=\int_{\left[\tau^{(i-1)} ; \tau^{(i)}\right)} h\left(\alpha_{i}, \beta_{i}, \gamma_{i}, t\right) d \mu(t) \quad \forall i=\overline{1, N-1}
$$

by

$$
H\left(\alpha_{N}, \beta_{N}, \gamma_{N}\right)=\int_{\left[\tau^{(N-1)} ; \tau^{(N)}\right]} h\left(\alpha_{N}, \beta_{N}, \gamma_{N}, t\right) d \mu(t) .
$$

Using (30) - (35), payoffs (8) - (10) in game (14) can be re-written as an interval-wise sum:

$$
\begin{gathered}
F(x(t), y(t), z(t))= \\
=\sum_{i=1}^{N-1} \int_{\left[\tau^{(i-1)} ; \tau^{(i)}\right)} f\left(\alpha_{i}, \beta_{i}, \gamma_{i}, t\right) d \mu(t)+\int_{\left[\tau^{(N-1)} ; \tau^{(N)}\right]} f\left(\alpha_{N}, \beta_{N}, \gamma_{N}, t\right) d \mu(t), \\
G(x(t), y(t), z(t))= \\
=\sum_{i=1}^{N-1} \int_{\left[\tau^{(i-1)} ; \tau^{(i)}\right)} g\left(\alpha_{i}, \beta_{i}, \gamma_{i}, t\right) d \mu(t)+\int_{\left[\tau^{(N-1)} ; \tau^{(N)}\right]} g\left(\alpha_{N}, \beta_{N}, \gamma_{N}, t\right) d \mu(t), \\
=\sum_{i=1}^{N-1} \int_{\left[\tau^{(i-1)} ; \tau^{(i)}\right)} h(x(t), y(t), z(t))= \\
\left.\int_{\left[\tau^{(N-1)} ; \tau^{(N)}\right]} h\left(\beta_{i}, \gamma_{i}, t\right) d \mu(t)+\beta_{N}, \gamma_{N}, t\right) d \mu(t) .
\end{gathered}
$$

In other words, if every equilibrium situation in pure strategies in game (14) on product (15) by conditions (1) - (13) is (or forced to be) of staircase functions satisfying conditions (16) - (26), then this game is equivalent to the succession of $N$ games (27) defined on parallelepiped (28) by (16) - (26) and (29) - (38). In this case game (14) can be represented by the succession of games (27).

Theorem 1. If each of $N$ games (27) by (16) - (26) and (29) - (38) has a single equilibrium situation in pure strategies, and game (14) on product (15) by conditions (1) - (13) is equivalent to the succession of these games, then the equilibrium situation in pure strategies in game (14) is determined by independently finding pure-strategy equilibria in $N$ games (27), whereupon these equilibria are successively stacked.

Proof. First, the equivalency means that game (14) has only staircase pure-strategy equilibria. Next, it should be proved that game (14) has a pure-strategy equilibrium 
situation, which is a successive stack of the $N$ "short" games (27). Let $\left\{\alpha_{i}^{*}, \beta_{i}^{*}, \gamma_{i}^{*}\right\}_{i=1}^{N}$ be pure-strategy equilibria in games (27) by (16) - (26) and (29) - (38). Then

$$
\begin{aligned}
& F\left(\alpha_{i}, \beta_{i}^{*}, \gamma_{i}^{*}\right) \leqslant F\left(\alpha_{i}^{*}, \beta_{i}^{*}, \gamma_{i}^{*}\right) \quad \forall \alpha_{i} \in\left[a_{\min } ; a_{\max }\right] \text { and } \forall i=\overline{1, N}, \\
& G\left(\alpha_{i}^{*}, \beta_{i}, \gamma_{i}^{*}\right) \leqslant G\left(\alpha_{i}^{*}, \beta_{i}^{*}, \gamma_{i}^{*}\right) \quad \forall \beta_{i} \in\left[b_{\min } ; b_{\text {max }}\right] \text { and } \forall i=\overline{1, N}, \\
& H\left(\alpha_{i}^{*}, \beta_{i}^{*}, \gamma_{i}\right) \leqslant H\left(\alpha_{i}^{*}, \beta_{i}^{*}, \gamma_{i}^{*}\right) \quad \forall \gamma_{i} \in\left[c_{\text {min }} ; c_{\text {max }}\right] \text { and } \forall i=\overline{1, N} .
\end{aligned}
$$

Inequalities (39) - (41) are re-written using statements (29) - (38):

$$
\begin{aligned}
& F\left(\alpha_{i}, \beta_{i}^{*}, \gamma_{i}^{*}\right)= \\
& =\int_{\left[\tau^{(i-1)} ; \tau^{(i)}\right)} f\left(\alpha_{i}, \beta_{i}^{*}, \gamma_{i}^{*}, t\right) d \mu(t) \leqslant \\
& \leqslant \int_{\left[\tau^{(i-1)} ; \tau^{(i)}\right)} f\left(\alpha_{i}^{*}, \beta_{i}^{*}, \gamma_{i}^{*}, t\right) d \mu(t)= \\
& =F\left(\alpha_{i}^{*}, \beta_{i}^{*}, \gamma_{i}^{*}\right) \quad \forall \alpha_{i} \in\left[a_{\min } ; a_{\max }\right] \text { and } \forall i=\overline{1, N-1} \text {, } \\
& F\left(\alpha_{N}, \beta_{N}^{*}, \gamma_{N}^{*}\right)= \\
& =\int_{\left[\tau^{(N-1)} ; \tau^{(N)}\right]} f\left(\alpha_{N}, \beta_{N}^{*}, \gamma_{N}^{*}, t\right) d \mu(t) \leqslant \\
& \leqslant \int_{\left[\tau^{(N-1)} ; \tau^{(N)}\right]} f\left(\alpha_{N}^{*}, \beta_{N}^{*}, \gamma_{N}^{*}, t\right) d \mu(t)= \\
& =F\left(\alpha_{N}^{*}, \beta_{N}^{*}, \gamma_{N}^{*}\right) \quad \forall \alpha_{N} \in\left[b_{\min } ; b_{\max }\right] \text {, } \\
& G\left(\alpha_{i}^{*}, \beta_{i}, \gamma_{i}^{*}\right)= \\
& =\int_{\left[\tau^{(i-1)} ; \tau^{(i)}\right)} g\left(\alpha_{i}^{*}, \beta_{i}, \gamma_{i}^{*}, t\right) d \mu(t) \leqslant \\
& \leqslant \int_{\left[\tau^{(i-1)} ; \tau^{(i)}\right)} g\left(\alpha_{i}^{*}, \beta_{i}^{*}, \gamma_{i}^{*}, t\right) d \mu(t)= \\
& =G\left(\alpha_{i}^{*}, \beta_{i}^{*}, \gamma_{i}^{*}\right) \quad \forall \beta_{i} \in\left[b_{\min } ; b_{\max }\right] \text { and } \forall i=\overline{1, N-1} \text {, } \\
& G\left(\alpha_{N}^{*}, \beta_{N}, \gamma_{N}^{*}\right)= \\
& =\int_{\left[\tau^{(N-1)} ; \tau^{(N)}\right]} g\left(\alpha_{N}^{*}, \beta_{N}, \gamma_{N}^{*}, t\right) d \mu(t) \leqslant
\end{aligned}
$$




$$
\begin{gathered}
\leqslant \int_{\left[\tau^{(N-1)} ; \tau^{(N)}\right]} g\left(\alpha_{N}^{*}, \beta_{N}^{*}, \gamma_{N}^{*}, t\right) d \mu(t)= \\
=G\left(\alpha_{N}^{*}, \beta_{N}^{*}, \gamma_{N}^{*}\right) \quad \forall \beta_{N} \in\left[b_{\min } ; b_{\max }\right], \\
H\left(\alpha_{i}^{*}, \beta_{i}^{*}, \gamma_{i}\right)= \\
=\int_{\left[\tau^{(i-1)} ; \tau^{(i)}\right)} h\left(\alpha_{i}^{*}, \beta_{i}^{*}, \gamma_{i}, t\right) d \mu(t) \leqslant \\
\leqslant \int_{\left[\tau^{(i-1)} ; \tau^{(i)}\right)} h\left(\alpha_{i}^{*}, \beta_{i}^{*}, \gamma_{i}^{*}, t\right) d \mu(t)= \\
\left.=\beta_{i}^{*}, \gamma_{i}^{*}\right) \forall \gamma_{i} \in\left[c_{\min } ; c_{\max }\right] \text { and } \forall i=\overline{1, N-1}, \\
=\int_{\left[\tau^{(N-1)} ; \tau^{(N)}\right]} h\left(\alpha_{N}^{*}, \beta_{N}^{*}, \gamma_{N}, t\right) d \mu(t) \leqslant \\
\leqslant \int_{\left[\tau^{(N-1)} ; \tau^{(N)}\right]} h\left(\alpha_{N}^{*}, \beta_{N}^{*}, \gamma_{N}^{*}, t\right) d \mu(t)= \\
=H\left(\alpha_{N}^{*}, \beta_{N}^{*}, \gamma_{N}^{*}\right) \quad \forall \gamma_{N} \in\left[c_{\min } ; c_{\max }^{*}\right] .
\end{gathered}
$$

So,

$$
\begin{aligned}
& \sum_{i=1}^{N} F\left(\alpha_{i}, \beta_{i}^{*}, \gamma_{i}^{*}\right) \leqslant \sum_{i=1}^{N} F\left(\alpha_{i}^{*}, \beta_{i}^{*}, \gamma_{i}^{*}\right), \\
& \sum_{i=1}^{N} G\left(\alpha_{i}^{*}, \beta_{i}, \gamma_{i}^{*}\right) \leqslant \sum_{i=1}^{N} G\left(\alpha_{i}^{*}, \beta_{i}^{*}, \gamma_{i}^{*}\right),
\end{aligned}
$$

and

$$
\sum_{i=1}^{N} H\left(\alpha_{i}^{*}, \beta_{i}^{*}, \gamma_{i}\right) \leqslant \sum_{i=1}^{N} H\left(\alpha_{i}^{*}, \beta_{i}^{*}, \gamma_{i}^{*}\right)
$$

Therefore, the successive stack of pure-strategy equilibria $\left\{\alpha_{i}^{*}, \beta_{i}^{*}, \gamma_{i}^{*}\right\}_{i=1}^{N}$ is a pure-strategy equilibrium in game (14). Obviously, games (27) can be solved independently, whose equilibria are stacked afterwards to form the pure-strategy equilibrium in game (14).

In fact, Theorem 1 claims that if each of $N$ "short" games (27) has a single purestrategy equilibrium, then the solution of three-person game (14) can be determined in a simpler way, by solving games (27) and successively stacking their equilibria. They are 
solved in parallel (independently), without caring of the succession. However, Theorem 1 does not determine a probability (likelihood) of the case when every "short" game has a single pure-strategy equilibrium. Obviously, the likelihood decays as the number of intervals increases.

Besides, Theorem 1 does not directly imply that the stacked equilibrium in game (14) is single. The question of whether the stacked equilibrium in game (14) is single or not is answered by the following assertion.

Theorem 2. If each of $N$ games (27) by (16) - (26) and (29) - (38) has a single equilibrium situation in pure strategies, and game (14) on product (15) by conditions (1) - (13) is equivalent to the succession of these games, then the equilibrium situation in pure strategies in game (14) is single being the successive stack of the "short" games equilibria.

Proof. The pure-strategy equilibrium in game (14) is constructed according to Theorem 1, i. e., it is the successive stack of pure-strategy equilibria $\left\{\alpha_{i}^{*}, \beta_{i}^{*}, \gamma_{i}^{*}\right\}_{i=1}^{N}$. Let this equilibrium be referred to as the

$$
\left\{\alpha_{i}^{*}, \beta_{i}^{*}, \gamma_{i}^{*}\right\}_{i=1}^{N} \text {-stack equilibrium. }
$$

Suppose that there is another pure-strategy equilibrium in game (14). First, let this equilibrium differ from (51) in just that the first player uses some $\alpha_{k}^{(0)} \in\left[a_{\min } ; a_{\text {max }}\right]$ instead of $\alpha_{k}^{*}$ by some $k \in\{\overline{1, N}\}$. So, this is the

$$
\left\{\left\{\alpha_{i}^{*}, \beta_{i}^{*}, \gamma_{i}^{*}\right\}_{i \in\{\overline{1, N}\} \backslash\{k\}} \cup\left\{\alpha_{k}^{(0)}, \beta_{k}^{*}, \gamma_{k}^{*}\right\}\right\} \text {-stack equilibrium, }
$$

which means that

$$
\begin{aligned}
& \sum_{i \in\{1, N\}\{\{k\}} F\left(\alpha_{i}, \beta_{i}^{*}, \gamma_{i}^{*}\right)+F\left(\alpha_{k}, \beta_{k}^{*}, \gamma_{k}^{*}\right) \leqslant \sum_{i \in\{1, N\}\{\{k\}} F\left(\alpha_{i}^{*}, \beta_{i}^{*}, \gamma_{i}^{*}\right)+F\left(\alpha_{k}^{(0)}, \beta_{k}^{*}, \gamma_{k}^{*}\right), \\
& \sum_{i \in\{1, N\}\{\{k\}} F\left(\alpha_{i}^{*}, \beta_{i}, \gamma_{i}^{*}\right)+G\left(\alpha_{k}^{(0)}, \beta_{k}, \gamma_{k}^{*}\right) \leqslant \sum_{i \in\{1, N\}\{k\}\}} F\left(\alpha_{i}^{*}, \beta_{i}^{*}, \gamma_{i}^{*}\right)+G\left(\alpha_{k}^{(0)}, \beta_{k}^{*}, \gamma_{k}^{*}\right), \\
& \sum_{i \in\{1, N\}\{k\}\}} H\left(\alpha_{i}^{*}, \beta_{i}^{*}, \gamma_{i}\right)+H\left(\alpha_{k}^{(0)}, \beta_{k}^{*}, \gamma_{k}\right) \leqslant \sum_{i \in\{1, N\}\{k\}\}} H\left(\alpha_{i}^{*}, \beta_{i}^{*}, \gamma_{i}^{*}\right)+H\left(\alpha_{k}^{(0)}, \beta_{k}^{*}, \gamma_{k}^{*}\right),
\end{aligned}
$$

i. e.,

$$
F\left(\alpha_{i}, \beta_{i}^{*}, \gamma_{i}^{*}\right) \leqslant F\left(\alpha_{i}^{*}, \beta_{i}^{*}, \gamma_{i}^{*}\right) \quad \forall \alpha_{i} \in\left[a_{\min } ; a_{\max }\right] \text { and } \forall i \in\{\overline{1, N}\} \backslash\{k\}
$$

by

$$
\begin{gathered}
F\left(\alpha_{k}, \beta_{k}^{*}, \gamma_{k}^{*}\right) \leqslant F\left(\alpha_{k}^{(0)}, \beta_{k}^{*}, \gamma_{k}^{*}\right) \quad \forall \alpha_{k} \in\left[a_{\min } ; a_{\max }\right], \\
G\left(\alpha_{i}^{*}, \beta_{i}, \gamma_{i}^{*}\right) \leqslant G\left(\alpha_{i}^{*}, \beta_{i}^{*}, \gamma_{i}^{*}\right) \quad \forall \beta_{i} \in\left[b_{\min } ; b_{\max }\right] \text { and } \forall i \in\{\overline{1, N}\} \backslash\{k\}
\end{gathered}
$$


by

$$
G\left(\alpha_{k}^{(0)}, \beta_{k}, \gamma_{k}^{*}\right) \leqslant G\left(\alpha_{k}^{(0)}, \beta_{k}^{*}, \gamma_{k}^{*}\right) \quad \forall \beta_{k} \in\left[b_{\min } ; b_{\max }\right]
$$

and

$$
H\left(\alpha_{i}^{*}, \beta_{i}^{*}, \gamma_{i}\right) \leqslant H\left(\alpha_{i}^{*}, \beta_{i}^{*}, \gamma_{i}^{*}\right) \quad \forall \gamma_{i} \in\left[c_{\min } ; c_{\max }\right] \text { and } \forall i \in\{\overline{1, N}\} \backslash\{k\}
$$

by

$$
H\left(\alpha_{k}^{(0)}, \beta_{k}^{*}, \gamma_{k}\right) \leqslant H\left(\alpha_{k}^{(0)}, \beta_{k}^{*}, \gamma_{k}^{*}\right) \quad \forall \gamma_{k} \in\left[c_{\min } ; c_{\max }\right]
$$

Inequalities (56), (58), and (60) imply that $\left\{\alpha_{k}^{(0)}, \beta_{k}^{*}, \gamma_{k}^{*}\right\}$ is a pure-strategy equilibrium at the $k$-th interval (in the $k$-th game), which is impossible due to every interval has a single pure-strategy equilibrium. The impossibility of the other pure-strategy equilibrium for the second and third players in such a case is proved symmetrically.

Second, suppose that the other pure-strategy equilibrium differs from (51) in that the first player uses some $\alpha_{k_{1}}^{(0)} \in\left[a_{\min } ; a_{\max }\right]$ instead of $\alpha_{k_{1}}^{*}$ by some $k_{1} \in\{\overline{1, N}\}$ and the second player uses some $\beta_{k_{2}}^{(0)} \in\left[b_{\min } ; b_{\max }\right]$ instead of $\beta_{k_{2}}^{*}$ by some $k_{2} \in\{\overline{1, N}\}$. So, this is the

$$
\left\{\left\{\alpha_{i}^{*}, \beta_{i}^{*}, \gamma_{i}^{*}\right\}_{i \in\{1, N\} \backslash\left\{k_{1}\right\}} \cup\left\{\alpha_{k_{1}}^{(0)}, \beta_{k_{1}}^{(0)}, \gamma_{k_{1}}^{*}\right\}\right\} \text {-stack equilibrium }
$$

if $k_{1}=k_{2}$, and is the

$$
\left\{\left\{\alpha_{i}^{*}, \beta_{i}^{*}, \gamma_{i}^{*}\right\}_{i \in\{1, N\} \backslash\left\{k_{1}, k_{2}\right\}} \cup\left\{\alpha_{k_{1}}^{(0)}, \beta_{k_{1}}^{*}, \gamma_{k_{1}}^{*}\right\} \cup\left\{\alpha_{k_{2}}^{*}, \beta_{k_{2}}^{(0)}, \gamma_{k_{2}}^{*}\right\}\right\} \text {-stack equilibrium }
$$

if $k_{1} \neq k_{2}$. Thus, (61) means that

$$
\sum_{i \in\{1, N\}\left\{k_{1}\right\}} F\left(\alpha_{i}, \beta_{i}^{*}, \gamma_{i}^{*}\right)+F\left(\alpha_{k_{1}}, \beta_{k_{1}}^{(0)}, \gamma_{k_{1}}^{*}\right) \leqslant \sum_{i \in\{1, N\}\left\{k_{1}\right\}} F\left(\alpha_{i}^{*}, \beta_{i}^{*}, \gamma_{i}^{*}\right)+F\left(\alpha_{k_{1}}^{(0)}, \beta_{k_{1}}^{(0)}, \gamma_{k_{1}}^{*}\right)
$$

and

$$
\sum_{i \in\{1, N\}\left\{k_{1}\right\}} G\left(\alpha_{i}^{*}, \beta_{i}, \gamma_{i}^{*}\right)+G\left(\alpha_{k_{1}}^{(0)}, \beta_{k_{1}}, \gamma_{k_{1}}^{*}\right) \leqslant \sum_{i \in\{1, N\}\left\{k_{1}\right\}} G\left(\alpha_{i}^{*}, \beta_{i}^{*}, \gamma_{i}^{*}\right)+G\left(\alpha_{k_{1}}^{(0)}, \beta_{k_{1}}^{(0)}, \gamma_{k_{1}}^{*}\right)
$$

and

$$
\sum_{i \in\{1, N\}\left\{k_{1}\right\}} H\left(\alpha_{i}^{*}, \beta_{i}^{*}, \gamma_{i}\right)+H\left(\alpha_{k_{1}}^{(0)}, \beta_{k_{1}}^{(0)}, \gamma_{k_{1}}\right) \leqslant \sum_{i \in\{1, N\}\left\{k_{1}\right\}} H\left(\alpha_{i}^{*}, \beta_{i}^{*}, \gamma_{i}^{*}\right)+H\left(\alpha_{k_{1}}^{(0)}, \beta_{k_{1}}^{(0)}, \gamma_{k_{1}}^{*}\right),
$$

i. e., inequalities (55) by $k=k_{1}$ and inequality

$$
F\left(\alpha_{k_{1}}, \beta_{k_{1}}^{(0)}, \gamma_{k_{1}}^{*}\right) \leqslant F\left(\alpha_{k_{1}}^{(0)}, \beta_{k_{1}}^{(0)}, \gamma_{k_{1}}^{*}\right) \quad \forall \alpha_{k_{1}} \in\left[a_{\min } ; a_{\max }\right]
$$

hold along with (39), inequalities (57) by $k=k_{1}$ and inequality 


$$
G\left(\alpha_{k_{1}}^{(0)}, \beta_{k_{1}}, \gamma_{k_{1}}^{*}\right) \leqslant G\left(\alpha_{k_{1}}^{(0)}, \beta_{k_{1}}^{(0)}, \gamma_{k_{1}}^{*}\right) \quad \forall \beta_{k_{1}} \in\left[b_{\min } ; b_{\max }\right]
$$

hold along with (40), inequalities (59) by $k=k_{1}$ and inequality

$$
H\left(\alpha_{k_{1}}^{(0)}, \beta_{k_{1}}^{(0)}, \gamma_{k_{1}}\right) \leqslant H\left(\alpha_{k_{1}}^{(0)}, \beta_{k_{1}}^{(0)}, \gamma_{k_{1}}^{*}\right) \quad \forall \gamma_{k_{1}} \in\left[c_{\min } ; c_{\max }\right]
$$

hold along with (41). Inequalities (66) - (68) imply that $\left\{\alpha_{k_{1}}^{(0)}, \beta_{k_{1}}^{(0)}, \gamma_{k_{1}}^{*}\right\}$ is a pure-strategy equilibrium at the $k_{1}$-th interval (in the $k_{1}$-th game), which is impossible. If (62) is true, then

$$
\begin{aligned}
& \sum_{i \in\{1, N\}\left\{k_{1}, k_{2}\right\}} F\left(\alpha_{i}, \beta_{i}^{*}, \gamma_{i}^{*}\right)+F\left(\alpha_{k_{1}}, \beta_{k_{1}}^{*}, \gamma_{k_{1}}^{*}\right)+F\left(\alpha_{k_{2}}, \beta_{k_{2}}^{(0)}, \gamma_{k_{2}}^{*}\right) \leqslant \\
\leqslant & \sum_{i \in\{1, N\}\}\left\{k_{1}, k_{2}\right\}} F\left(\alpha_{i}^{*}, \beta_{i}^{*}, \gamma_{i}^{*}\right)+F\left(\alpha_{k_{1}}^{(0)}, \beta_{k_{1}}^{*}, \gamma_{k_{1}}^{*}\right)+F\left(\alpha_{k_{2}}^{*}, \beta_{k_{2}}^{(0)}, \gamma_{k_{2}}^{*}\right)
\end{aligned}
$$

and

$$
\begin{aligned}
& \sum_{i \in\{1, N\}\left\{\left\{k_{1}, k_{2}\right\}\right.} G\left(\alpha_{i}^{*}, \beta_{i}, \gamma_{i}^{*}\right)+G\left(\alpha_{k_{1}}^{(0)}, \beta_{k_{1}}, \gamma_{k_{1}}^{*}\right)+G\left(\alpha_{k_{2}}^{*}, \beta_{k_{2}}, \gamma_{k_{2}}^{*}\right) \leqslant \\
\leqslant & \sum_{i \in\{1, N\} \backslash\left\{k_{1}, k_{2}\right\}} G\left(\alpha_{i}^{*}, \beta_{i}^{*}, \gamma_{i}^{*}\right)+G\left(\alpha_{k_{1}}^{(0)}, \beta_{k_{1}}^{*}, \gamma_{k_{1}}^{*}\right)+G\left(\alpha_{k_{2}}^{*}, \beta_{k_{2}}^{(0)}, \gamma_{k_{2}}^{*}\right)
\end{aligned}
$$

and

$$
\begin{aligned}
& \sum_{i \in\{1, N\}\left\{\left\{k_{1}, k_{2}\right\}\right.} H\left(\alpha_{i}^{*}, \beta_{i}^{*}, \gamma_{i}\right)+H\left(\alpha_{k_{1}}^{(0)}, \beta_{k_{1}}^{*}, \gamma_{k_{1}}\right)+H\left(\alpha_{k_{2}}^{*}, \beta_{k_{2}}^{(0)}, \gamma_{k_{2}}\right) \leqslant \\
& \leqslant \sum_{i \in\{1, N\} \backslash\left\{k_{1}, k_{2}\right\}} H\left(\alpha_{i}^{*}, \beta_{i}^{*}, \gamma_{i}^{*}\right)+H\left(\alpha_{k_{1}}^{(0)}, \beta_{k_{1}}^{*}, \gamma_{k_{1}}^{*}\right)+H\left(\alpha_{k_{2}}^{*}, \beta_{k_{2}}^{(0)}, \gamma_{k_{2}}^{*}\right),
\end{aligned}
$$

i. e., inequalities

$$
F\left(\alpha_{i}, \beta_{i}^{*}, \gamma_{i}^{*}\right) \leqslant F\left(\alpha_{i}^{*}, \beta_{i}^{*}, \gamma_{i}^{*}\right) \quad \forall \alpha_{i} \in\left[a_{\min } ; a_{\max }\right] \text { and } \forall i \in\{\overline{1, N}\} \backslash\left\{k_{1}, k_{2}\right\}
$$

and inequality

$$
\begin{gathered}
F\left(\alpha_{k_{1}}, \beta_{k_{1}}^{*}, \gamma_{k_{1}}^{*}\right)+F\left(\alpha_{k_{2}}, \beta_{k_{2}}^{(0)}, \gamma_{k_{2}}^{*}\right) \leqslant F\left(\alpha_{k_{1}}^{(0)}, \beta_{k_{1}}^{*}, \gamma_{k_{1}}^{*}\right)+F\left(\alpha_{k_{2}}^{*}, \beta_{k_{2}}^{(0)}, \gamma_{k_{2}}^{*}\right) \\
\forall \alpha_{k_{1}} \in\left[a_{\min } ; a_{\max }\right] \text { and } \forall \alpha_{k_{2}} \in\left[a_{\min } ; a_{\max }\right]
\end{gathered}
$$

hold along with (39), inequalities

$$
G\left(\alpha_{i}^{*}, \beta_{i}, \gamma_{i}^{*}\right) \leqslant G\left(\alpha_{i}^{*}, \beta_{i}^{*}, \gamma_{i}^{*}\right) \quad \forall \beta_{i} \in\left[b_{\min } ; b_{\max }\right] \text { and } \forall i \in\{\overline{1, N}\} \backslash\left\{k_{1}, k_{2}\right\}
$$

and inequality

$$
\begin{gathered}
G\left(\alpha_{k_{1}}^{(0)}, \beta_{k_{1}}, \gamma_{k_{1}}^{*}\right)+G\left(\alpha_{k_{2}}^{*}, \beta_{k_{2}}, \gamma_{k_{2}}^{*}\right) \leqslant G\left(\alpha_{k_{1}}^{(0)}, \beta_{k_{1}}^{*}, \gamma_{k_{1}}^{*}\right)+G\left(\alpha_{k_{2}}^{*}, \beta_{k_{2}}^{(0)}, \gamma_{k_{2}}^{*}\right) \\
\forall \beta_{k_{1}} \in\left[b_{\min } ; b_{\max }\right] \text { and } \forall \beta_{k_{2}} \in\left[b_{\min } ; b_{\max }\right]
\end{gathered}
$$


hold along with (40), inequalities

$$
H\left(\alpha_{i}^{*}, \beta_{i}^{*}, \gamma_{i}\right) \leqslant H\left(\alpha_{i}^{*}, \beta_{i}^{*}, \gamma_{i}^{*}\right) \quad \forall \gamma_{i} \in\left[c_{\min } ; c_{\max }\right] \text { and } \forall i \in\{\overline{1, N}\} \backslash\left\{k_{1}, k_{2}\right\}
$$

and inequality

$$
\begin{gathered}
H\left(\alpha_{k_{1}}^{(0)}, \beta_{k_{1}}^{*}, \gamma_{k_{1}}\right)+H\left(\alpha_{k_{2}}^{*}, \beta_{k_{2}}^{(0)}, \gamma_{k_{2}}\right) \leqslant H\left(\alpha_{k_{1}}^{(0)}, \beta_{k_{1}}^{*}, \gamma_{k_{1}}^{*}\right)+H\left(\alpha_{k_{2}}^{*}, \beta_{k_{2}}^{(0)}, \gamma_{k_{2}}^{*}\right) \\
\forall \gamma_{k_{1}} \in\left[c_{\min } ; c_{\max }\right] \text { and } \forall \gamma_{k_{2}} \in\left[c_{\min } ; c_{\max }\right]
\end{gathered}
$$

hold along with (41). Plugging $\alpha_{k_{2}}=\alpha_{k_{2}}^{*}$ in the left side of inequality (73) and plugging $\beta_{k_{2}}=\beta_{k_{2}}^{(0)}$ in the left side of inequality (75) and plugging $\gamma_{k_{2}}=\gamma_{k_{2}}^{*}$ in the left side of inequality (77) gives inequalities (56), (58), (60) for $k=k_{1}$, which are impossible due to $\left\{\alpha_{k_{1}}^{(0)}, \beta_{k_{1}}^{*}, \gamma_{k_{1}}^{*}\right\}$ is not a pure-strategy equilibrium. Therefore, the supposition about (61) and (62) are true is contradictory.

Third, suppose that the other pure-strategy equilibrium differs from (51) in that the first player uses some $\alpha_{k_{1}}^{(0)} \in\left[a_{\min } ; a_{\text {max }}\right]$ instead of $\alpha_{k_{1}}^{*}$ by some $k_{1} \in\{\overline{1, N}\}$, the second player uses some $\beta_{k_{2}}^{(0)} \in\left[b_{\min } ; b_{\max }\right]$ instead of $\beta_{k_{2}}^{*}$ by some $k_{2} \in\{\overline{1, N}\}$, and the third player uses some $\gamma_{k_{3}}^{(0)} \in\left[c_{\min } ; c_{\max }\right]$ instead of $\gamma_{k_{3}}^{*}$ by some $k_{3} \in\{\overline{1, N}\}$. So, this is the

$$
\left\{\left\{\alpha_{i}^{*}, \beta_{i}^{*}, \gamma_{i}^{*}\right\}_{i \in\{\overline{1, N}\}\left\{k_{1}, k_{2}, k_{3}\right\}} \cup\left\{\alpha_{k_{1}}^{(0)}, \beta_{k_{1}}^{*}, \gamma_{k_{1}}^{*}\right\} \cup\left\{\alpha_{k_{2}}^{*}, \beta_{k_{2}}^{(0)}, \gamma_{k_{2}}^{*}\right\} \cup\left\{\alpha_{k_{3}}^{*}, \beta_{k_{3}}^{*}, \gamma_{k_{3}}^{(0)}\right\}\right\}
$$

-stack equilibrium.

Thus, (78) means that

$$
\begin{gathered}
\sum_{i \in\{1, N\} \backslash\left\{k_{1}, k_{2}, k_{3}\right\}} F\left(\alpha_{i}, \beta_{i}^{*}, \gamma_{i}^{*}\right)+ \\
+F\left(\alpha_{k_{1}}, \beta_{k_{1}}^{*}, \gamma_{k_{1}}^{*}\right)+F\left(\alpha_{k_{2}}, \beta_{k_{2}}^{(0)}, \gamma_{k_{2}}^{*}\right)+F\left(\alpha_{k_{3}}, \beta_{k_{3}}^{*}, \gamma_{k_{3}}^{(0)}\right) \leqslant \\
\leqslant \sum_{i \in\{1, N\} \backslash\left\{k_{1}, k_{2}, k_{3}\right\}} F\left(\alpha_{i}^{*}, \beta_{i}^{*}, \gamma_{i}^{*}\right)+ \\
+F\left(\alpha_{k_{1}}^{(0)}, \beta_{k_{1}}^{*}, \gamma_{k_{1}}^{*}\right)+F\left(\alpha_{k_{2}}^{*}, \beta_{k_{2}}^{(0)}, \gamma_{k_{2}}^{*}\right)+F\left(\alpha_{k_{3}}^{*}, \beta_{k_{3}}^{*}, \gamma_{k_{3}}^{(0)}\right)
\end{gathered}
$$

and

$$
\begin{gathered}
\sum_{i \in\{1, N\}\left\{\left\{k_{1}, k_{2}, k_{3}\right\}\right.} G\left(\alpha_{i}^{*}, \beta_{i}, \gamma_{i}^{*}\right)+ \\
+G\left(\alpha_{k_{1}}^{(0)}, \beta_{k_{1}}, \gamma_{k_{1}}^{*}\right)+G\left(\alpha_{k_{2}}^{*}, \beta_{k_{2}}, \gamma_{k_{2}}^{*}\right)+G\left(\alpha_{k_{3}}^{*}, \beta_{k_{3}}, \gamma_{k_{3}}^{(0)}\right) \leqslant \\
\leqslant \sum_{i \in\{1, N\} \backslash\left\{k_{1}, k_{2}, k_{3}\right\}} G\left(\alpha_{i}^{*}, \beta_{i}^{*}, \gamma_{i}^{*}\right)+ \\
+G\left(\alpha_{k_{1}}^{(0)}, \beta_{k_{1}}^{*}, \gamma_{k_{1}}^{*}\right)+G\left(\alpha_{k_{2}}^{*}, \beta_{k_{2}}^{(0)}, \gamma_{k_{2}}^{*}\right)+G\left(\alpha_{k_{3}}^{*}, \beta_{k_{3}}^{*}, \gamma_{k_{3}}^{(0)}\right)
\end{gathered}
$$


and

$$
\begin{gathered}
\sum_{i \in\{1, N\}\left\{\left\{k_{1}, k_{2}, k_{3}\right\}\right.} H\left(\alpha_{i}^{*}, \beta_{i}^{*}, \gamma_{i}\right)+ \\
+H\left(\alpha_{k_{1}}^{(0)}, \beta_{k_{1}}^{*}, \gamma_{k_{1}}\right)+H\left(\alpha_{k_{2}}^{*}, \beta_{k_{2}}^{(0)}, \gamma_{k_{2}}\right)+H\left(\alpha_{k_{3}}^{*}, \beta_{k_{3}}^{*}, \gamma_{k_{3}}\right) \leqslant \\
\leqslant \sum_{i \in\{1, N\} \backslash\left\{k_{1}, k_{2}, k_{3}\right\}} H\left(\alpha_{i}^{*}, \beta_{i}^{*}, \gamma_{i}^{*}\right)+ \\
+H\left(\alpha_{k_{1}}^{(0)}, \beta_{k_{1}}^{*}, \gamma_{k_{1}}^{*}\right)+H\left(\alpha_{k_{2}}^{*}, \beta_{k_{2}}^{(0)}, \gamma_{k_{2}}^{*}\right)+H\left(\alpha_{k_{3}}^{*}, \beta_{k_{3}}^{*}, \gamma_{k_{3}}^{(0)}\right),
\end{gathered}
$$

i. e., inequalities

$$
F\left(\alpha_{i}, \beta_{i}^{*}, \gamma_{i}^{*}\right) \leqslant F\left(\alpha_{i}^{*}, \beta_{i}^{*}, \gamma_{i}^{*}\right) \quad \forall \alpha_{i} \in\left[a_{\min } ; a_{\max }\right] \text { and } \forall i \in\{\overline{1, N}\} \backslash\left\{k_{1}, k_{2}, k_{3}\right\}
$$

and inequality

$$
\begin{gathered}
F\left(\alpha_{k_{1}}, \beta_{k_{1}}^{*}, \gamma_{k_{1}}^{*}\right)+F\left(\alpha_{k_{2}}, \beta_{k_{2}}^{(0)}, \gamma_{k_{2}}^{*}\right)+F\left(\alpha_{k_{3}}, \beta_{k_{3}}^{*}, \gamma_{k_{3}}^{(0)}\right) \leqslant \\
\leqslant F\left(\alpha_{k_{1}}^{(0)}, \beta_{k_{1}}^{*}, \gamma_{k_{1}}^{*}\right)+F\left(\alpha_{k_{2}}^{*}, \beta_{k_{2}}^{(0)}, \gamma_{k_{2}}^{*}\right)+F\left(\alpha_{k_{3}}^{*}, \beta_{k_{3}}^{*}, \gamma_{k_{3}}^{(0)}\right) \\
\forall \alpha_{k_{1}} \in\left[a_{\min } ; a_{\max }\right] \text { and } \forall \alpha_{k_{2}} \in\left[a_{\min } ; a_{\max }\right] \text { and } \forall \alpha_{k_{3}} \in\left[a_{\min } ; a_{\max }\right]
\end{gathered}
$$

hold along with (39), inequalities

$$
G\left(\alpha_{i}^{*}, \beta_{i}, \gamma_{i}^{*}\right) \leqslant G\left(\alpha_{i}^{*}, \beta_{i}^{*}, \gamma_{i}^{*}\right) \quad \forall \beta_{i} \in\left[b_{\text {min }} ; b_{\text {max }}\right] \text { and } \forall i \in\{\overline{1, N}\} \backslash\left\{k_{1}, k_{2}, k_{3}\right\}
$$

and inequality

$$
\begin{gathered}
G\left(\alpha_{k_{1}}^{(0)}, \beta_{k_{1}}, \gamma_{k_{1}}^{*}\right)+G\left(\alpha_{k_{2}}^{*}, \beta_{k_{2}}, \gamma_{k_{2}}^{*}\right)+G\left(\alpha_{k_{3}}^{*}, \beta_{k_{3}}, \gamma_{k_{3}}^{(0)}\right) \leqslant \\
\leqslant G\left(\alpha_{k_{1}}^{(0)}, \beta_{k_{1}}^{*}, \gamma_{k_{1}}^{*}\right)+G\left(\alpha_{k_{2}}^{*}, \beta_{k_{2}}^{(0)}, \gamma_{k_{2}}^{*}\right)+G\left(\alpha_{k_{3}}^{*}, \beta_{k_{3}}^{*}, \gamma_{k_{3}}^{(0)}\right) \\
\forall \beta_{k_{1}} \in\left[b_{\min } ; b_{\max }\right] \text { and } \forall \beta_{k_{2}} \in\left[b_{\min } ; b_{\max }\right] \text { and } \forall \beta_{k_{3}} \in\left[b_{\min } ; b_{\max }\right]
\end{gathered}
$$

hold along with (40), inequalities

$$
H\left(\alpha_{i}^{*}, \beta_{i}^{*}, \gamma_{i}\right) \leqslant H\left(\alpha_{i}^{*}, \beta_{i}^{*}, \gamma_{i}^{*}\right) \quad \forall \gamma_{i} \in\left[c_{\min } ; c_{\max }\right] \text { and } \forall i \in\{\overline{1, N}\} \backslash\left\{k_{1}, k_{2}, k_{3}\right\}
$$

and inequality

$$
\begin{gathered}
H\left(\alpha_{k_{1}}^{(0)}, \beta_{k_{1}}^{*}, \gamma_{k_{1}}\right)+H\left(\alpha_{k_{2}}^{*}, \beta_{k_{2}}^{(0)}, \gamma_{k_{2}}\right)+H\left(\alpha_{k_{3}}^{*}, \beta_{k_{3}}^{*}, \gamma_{k_{3}}\right) \leqslant \\
\leqslant H\left(\alpha_{k_{1}}^{(0)}, \beta_{k_{1}}^{*}, \gamma_{k_{1}}^{*}\right)+H\left(\alpha_{k_{2}}^{*}, \beta_{k_{2}}^{(0)}, \gamma_{k_{2}}^{*}\right)+H\left(\alpha_{k_{3}}^{*}, \beta_{k_{3}}^{*}, \gamma_{k_{3}}^{(0)}\right) \\
\forall \gamma_{k_{1}} \in\left[c_{\min } ; c_{\max }\right] \text { and } \forall \gamma_{k_{2}} \in\left[c_{\min } ; c_{\max }\right] \text { and } \forall \gamma_{k_{3}} \in\left[c_{\min } ; c_{\max }\right]
\end{gathered}
$$

hold along with (41). Plugging $\alpha_{k_{2}}=\alpha_{k_{2}}^{*}$ and $\alpha_{k_{3}}=\alpha_{k_{3}}^{*}$ in the left side of inequality (83) gives inequality

$$
F\left(\alpha_{k_{1}}, \beta_{k_{1}}^{*}, \gamma_{k_{1}}^{*}\right) \leqslant F\left(\alpha_{k_{1}}^{(0)}, \beta_{k_{1}}^{*}, \gamma_{k_{1}}^{*}\right) \quad \forall \alpha_{k_{1}} \in\left[a_{\min } ; a_{\max }\right]
$$


plugging $\beta_{k_{2}}=\beta_{k_{2}}^{(0)}$ and $\beta_{k_{3}}=\beta_{k_{3}}^{*}$ in the left side of inequality (85) gives inequality

$$
G\left(\alpha_{k_{1}}^{(0)}, \beta_{k_{1}}, \gamma_{k_{1}}^{*}\right) \leqslant G\left(\alpha_{k_{1}}^{(0)}, \beta_{k_{1}}^{*}, \gamma_{k_{1}}^{*}\right) \quad \forall \beta_{k_{1}} \in\left[b_{\min } ; b_{\max }\right]
$$

and plugging $\gamma_{k_{2}}=\gamma_{k_{2}}^{*}$ and $\gamma_{k_{3}}=\gamma_{k_{3}}^{(0)}$ in the left side of inequality (87) gives inequality

$$
H\left(\alpha_{k_{1}}^{(0)}, \beta_{k_{1}}^{*}, \gamma_{k_{1}}\right) \leqslant H\left(\alpha_{k_{1}}^{(0)}, \beta_{k_{1}}^{*}, \gamma_{k_{1}}^{*}\right) \quad \forall \gamma_{k_{1}} \in\left[c_{\min } ; c_{\max }\right]
$$

Inequalities (88) - (90) imply that $\left\{\alpha_{k_{1}}^{(0)}, \beta_{k_{1}}^{*}, \gamma_{k_{1}}^{*}\right\}$ is a pure-strategy equilibrium, which is impossible. Therefore, (78) is false.

Next, suppose that the other pure-strategy equilibrium differs from (51) in that the first player uses some $\alpha_{k_{1}}^{(0)} \in\left[a_{\min } ; a_{\max }\right]$ instead of $\alpha_{k_{1}}^{*}$ by some $k_{1} \in\{\overline{1, N}\}$ and some $\alpha_{k_{2}}^{(0)} \in\left[a_{\min } ; a_{\max }\right]$ instead of $\alpha_{k_{2}}^{*}$ by some $k_{2} \in\{\overline{1, N}\}$. The respective

$$
\left\{\left\{\alpha_{i}^{*}, \beta_{i}^{*}, \gamma_{i}^{*}\right\}_{i \in\{1, N\}\left\{k_{1}, k_{2}\right\}} \cup\left\{\alpha_{k_{1}}^{(0)}, \beta_{k_{1}}^{*}, \gamma_{k_{1}}^{*}\right\} \bigcup\left\{\alpha_{k_{2}}^{(0)}, \beta_{k_{2}}^{*}, \gamma_{k_{2}}^{*}\right\}\right\} \text {-stack equilibrium }
$$

means that

$$
\begin{aligned}
& \sum_{i \in\{1, N\}\left\{k_{1}, k_{2}\right\}} F\left(\alpha_{i}, \beta_{i}^{*}, \gamma_{i}^{*}\right)+F\left(\alpha_{k_{1}}, \beta_{k_{1}}^{*}, \gamma_{k_{1}}^{*}\right)+F\left(\alpha_{k_{2}}, \beta_{k_{2}}^{*}, \gamma_{k_{2}}^{*}\right) \leqslant \\
\leqslant & \sum_{i \in\{1, N\}\left\{\left\{k_{1}, k_{2}\right\}\right.} F\left(\alpha_{i}^{*}, \beta_{i}^{*}, \gamma_{i}^{*}\right)+F\left(\alpha_{k_{1}}^{(0)}, \beta_{k_{1}}^{*}, \gamma_{k_{1}}^{*}\right)+F\left(\alpha_{k_{2}}^{(0)}, \beta_{k_{2}}^{*}, \gamma_{k_{2}}^{*}\right)
\end{aligned}
$$

and

$$
\begin{aligned}
& \sum_{i \in\{1, N\}\left\{\left\{k_{1}, k_{2}\right\}\right.} G\left(\alpha_{i}^{*}, \beta_{i}, \gamma_{i}^{*}\right)+G\left(\alpha_{k_{1}}^{(0)}, \beta_{k_{1}}, \gamma_{k_{1}}^{*}\right)+G\left(\alpha_{k_{2}}^{(0)}, \beta_{k_{2}}, \gamma_{k_{2}}^{*}\right) \leqslant \\
\leqslant & \sum_{i \in\{1, N\}\}\left\{k_{1}, k_{2}\right\}} G\left(\alpha_{i}^{*}, \beta_{i}^{*}, \gamma_{i}^{*}\right)+G\left(\alpha_{k_{1}}^{(0)}, \beta_{k_{1}}^{*}, \gamma_{k_{1}}^{*}\right)+G\left(\alpha_{k_{2}}^{(0)}, \beta_{k_{2}}^{*}, \gamma_{k_{2}}^{*}\right)
\end{aligned}
$$

and

$$
\begin{aligned}
& \sum_{i \in\{1, N\}\left\{\left\{k_{1}, k_{2}\right\}\right.} H\left(\alpha_{i}^{*}, \beta_{i}^{*}, \gamma_{i}\right)+H\left(\alpha_{k_{1}}^{(0)}, \beta_{k_{1}}^{*}, \gamma_{k_{1}}\right)+H\left(\alpha_{k_{2}}^{(0)}, \beta_{k_{2}}^{*}, \gamma_{k_{2}}\right) \leqslant \\
& \leqslant \sum_{i \in\{1, N\} \backslash\left\{k_{1}, k_{2}\right\}} H\left(\alpha_{i}^{*}, \beta_{i}^{*}, \gamma_{i}^{*}\right)+H\left(\alpha_{k_{1}}^{(0)}, \beta_{k_{1}}^{*}, \gamma_{k_{1}}^{*}\right)+H\left(\alpha_{k_{2}}^{(0)}, \beta_{k_{2}}^{*}, \gamma_{k_{2}}^{*}\right),
\end{aligned}
$$

i. e., inequalities (72) and inequality

$$
\begin{gathered}
F\left(\alpha_{k_{1}}, \beta_{k_{1}}^{*}, \gamma_{k_{1}}^{*}\right)+F\left(\alpha_{k_{2}}, \beta_{k_{2}}^{*}, \gamma_{k_{2}}^{*}\right) \leqslant F\left(\alpha_{k_{1}}^{(0)}, \beta_{k_{1}}^{*}, \gamma_{k_{1}}^{*}\right)+F\left(\alpha_{k_{2}}^{(0)}, \beta_{k_{2}}^{*}, \gamma_{k_{2}}^{*}\right) \\
\forall \alpha_{k_{1}} \in\left[a_{\min } ; a_{\max }\right] \text { and } \forall \alpha_{k_{2}} \in\left[a_{\min } ; a_{\max }\right]
\end{gathered}
$$

hold along with (39), inequalities (74) and inequality 


$$
\begin{gathered}
G\left(\alpha_{k_{1}}^{(0)}, \beta_{k_{1}}, \gamma_{k_{1}}^{*}\right)+G\left(\alpha_{k_{2}}^{(0)}, \beta_{k_{2}}, \gamma_{k_{2}}^{*}\right) \leqslant G\left(\alpha_{k_{1}}^{(0)}, \beta_{k_{1}}^{*}, \gamma_{k_{1}}^{*}\right)+G\left(\alpha_{k_{2}}^{(0)}, \beta_{k_{2}}^{*}, \gamma_{k_{2}}^{*}\right) \\
\forall \beta_{k_{1}} \in\left[b_{\min } ; b_{\max }\right] \text { and } \forall \beta_{k_{2}} \in\left[b_{\min } ; b_{\max }\right]
\end{gathered}
$$

hold along with (40), inequalities (76) and inequality

$$
\begin{gathered}
H\left(\alpha_{k_{1}}^{(0)}, \beta_{k_{1}}^{*}, \gamma_{k_{1}}\right)+H\left(\alpha_{k_{2}}^{(0)}, \beta_{k_{2}}^{*}, \gamma_{k_{2}}\right) \leqslant H\left(\alpha_{k_{1}}^{(0)}, \beta_{k_{1}}^{*}, \gamma_{k_{1}}^{*}\right)+H\left(\alpha_{k_{2}}^{(0)}, \beta_{k_{2}}^{*}, \gamma_{k_{2}}^{*}\right) \\
\forall \gamma_{k_{1}} \in\left[c_{\min } ; c_{\max }\right] \text { and } \forall \gamma_{k_{2}} \in\left[c_{\min } ; c_{\max }\right]
\end{gathered}
$$

hold along with (41). Plugging $\alpha_{k_{2}}=\alpha_{k_{2}}^{(0)}$ in the left side of inequality (95) and plugging $\beta_{k_{2}}=\beta_{k_{2}}^{*}$ in the left side of inequality (96) and plugging $\gamma_{k_{2}}=\gamma_{k_{2}}^{*}$ in the left side of inequality (97) gives inequalities (88) - (90), which are impossible due to $\left\{\alpha_{k_{1}}^{(0)}, \beta_{k_{1}}^{*}, \gamma_{k_{1}}^{*}\right\}$ is not a pure-strategy equilibrium. So, the supposition about (91) is contradictory. The impossibility of the other pure-strategy equilibrium for the second and third players in such a case (of two intervals) is proved symmetrically. The impossibility of other pure-strategy equilibria differing from (51) in that the players use some other values at intervals is proved symmetrically as well.

Therefore, Theorem 2 along with Theorem 1 allows obtaining the single pure-strategy solution of game (14) directly from equilibria in games (27). The application of these assertions significantly simplifies the solving of game (14). Under conditions of the assertions, game (14) is "discretized" or "broken" into simpler three-person games, whereupon their equilibria are stacked.

But what if the conditions are inverted? Does the equilibrium singularity in games (27) change when the single pure-strategy equilibrium of game (14) is already known? This question is answered by the following assertion.

Theorem 3. If game (14) on product (15) by conditions (1) - (13) and (16) - (26) has a single equilibrium situation in pure strategies, then each of $N$ games (27) by (16) - (26) and (29) - (38) has a single pure-strategy equilibrium, which is the respective interval part of the game (14) equilibrium.

Proof. Let game (14) have (51) which is single. This implies that inequalities (48) - (50) hold. Plugging

$$
\alpha_{i}=\alpha_{i}^{*}, \beta_{i}=\beta_{i}^{*}, \gamma_{i}=\gamma_{i}^{*} \forall i \in\{\overline{1, N}\} \backslash\left\{k_{*}\right\}
$$

in the left side of inequality (48), (49), (50), respectively, gives inequalities

$$
F\left(\alpha_{k_{*}}, \beta_{k_{*}}^{*}, \gamma_{k_{*}}^{*}\right) \leqslant F\left(\alpha_{k_{*}}^{*}, \beta_{k_{*}}^{*}, \gamma_{k_{*}}^{*}\right) \quad \forall \alpha_{k_{*}} \in\left[a_{\min } ; a_{\max }\right]
$$

and

$$
G\left(\alpha_{k_{*}}^{*}, \beta_{k_{*}}, \gamma_{k_{*}}^{*}\right) \leqslant G\left(\alpha_{k_{*}}^{*}, \beta_{k_{*}}^{*}, \gamma_{k_{*}}^{*}\right) \quad \forall \beta_{k_{*}} \in\left[b_{\min } ; b_{\max }\right]
$$

and

$$
H\left(\alpha_{k_{s}}^{*}, \beta_{k_{*}}^{*}, \gamma_{k_{*}}\right) \leqslant H\left(\alpha_{k_{*}}^{*}, \beta_{k_{*}}^{*}, \gamma_{k_{*}}^{*}\right) \quad \forall \gamma_{k_{*}} \in\left[c_{\min } ; c_{\max }\right]
$$


whence $\left\{\alpha_{k_{*}}^{*}, \beta_{k_{*}}^{*}, \gamma_{k_{*}}^{*}\right\}$ is a pure-strategy equilibrium at the $k_{*}$-th interval (in the $k_{*}$-th game) for every $k_{*} \in\{\overline{1, N}\}$.

Suppose that $\exists k_{0} \in\{\overline{1, N}\}$ such that $\left\{\alpha_{k_{0}}^{(0)}, \beta_{k_{0}}^{*}, \gamma_{k_{0}}^{*}\right\}$ is an equilibrium by $\alpha_{k_{0}}^{(0)} \neq \alpha_{k_{0}}^{*}$. Then inequalities

$$
F\left(\alpha_{k_{0}}, \beta_{k_{0}}^{*}, \gamma_{k_{0}}^{*}\right) \leqslant F\left(\alpha_{k_{0}}^{(0)}, \beta_{k_{0}}^{*}, \gamma_{k_{0}}^{*}\right) \quad \forall \alpha_{k_{0}} \in\left[a_{\min } ; a_{\max }\right]
$$

and

$$
G\left(\alpha_{k_{0}}^{(0)}, \beta_{k_{0}}, \gamma_{k_{0}}^{*}\right) \leqslant G\left(\alpha_{k_{0}}^{(0)}, \beta_{k_{0}}^{*}, \gamma_{k_{0}}^{*}\right) \quad \forall \beta_{k_{0}} \in\left[b_{\min } ; b_{\max }\right]
$$

and

$$
H\left(\alpha_{k_{0}}^{(0)}, \beta_{k_{0}}^{*}, \gamma_{k_{0}}\right) \leqslant H\left(\alpha_{k_{0}}^{(0)}, \beta_{k_{0}}^{*}, \gamma_{k_{0}}^{*}\right) \quad \forall \gamma_{k_{0}} \in\left[c_{\min } ; c_{\max }\right]
$$

hold, whence inequalities

$$
\begin{aligned}
& \sum_{k_{*}\left\{\{1, N\}\left\{k_{0}\right\}\right.} F\left(\alpha_{k_{*}}, \beta_{k_{*}}^{*}, \gamma_{k_{*}}^{*}\right)+F\left(\alpha_{k_{0}}, \beta_{k_{0}}^{*}, \gamma_{k_{0}}^{*}\right) \leqslant \\
\leqslant & \sum_{k_{*} \in\{1, N\}\left\{k_{0}\right\}} F\left(\alpha_{k_{*}}^{*}, \beta_{k_{*}}^{*}, \gamma_{k_{*}}^{*}\right)+F\left(\alpha_{k_{0}}^{(0)}, \beta_{k_{0}}^{*}, \gamma_{k_{0}}^{*}\right)
\end{aligned}
$$

and

$$
\begin{aligned}
& \sum_{k_{*} \in\{1, N\}\left\{\left\{k_{0}\right\}\right.} G\left(\alpha_{k_{*}}^{*}, \beta_{k_{*}}, \gamma_{k_{*}}^{*}\right)+G\left(\alpha_{k_{0}}^{(0)}, \beta_{k_{0}}, \gamma_{k_{0}}^{*}\right) \leqslant \\
\leqslant & \sum_{k_{*}\left\{\{ 1 , N \} \left\{\left\{k_{0}\right\}\right.\right.} G\left(\alpha_{k_{*}}^{*}, \beta_{k_{*}}^{*}, \gamma_{k_{*}}^{*}\right)+G\left(\alpha_{k_{0}}^{(0)}, \beta_{k_{0}}^{*}, \gamma_{k_{0}}^{*}\right)
\end{aligned}
$$

and

$$
\begin{aligned}
& \sum_{k_{*} \in\{1, N\}\left\{k_{0}\right\}} H\left(\alpha_{k_{*}}^{*}, \beta_{k_{*}}^{*}, \gamma_{k_{*}}\right)+H\left(\alpha_{k_{0}}^{(0)}, \beta_{k_{0}}^{*}, \gamma_{k_{0}}\right) \leqslant \\
& \leqslant \sum_{\left.k_{*} \in\{1, N\}\right\}\left\{k_{0}\right\}} H\left(\alpha_{k_{*}}^{*}, \beta_{k_{*}}^{*}, \gamma_{k_{*}}^{*}\right)+H\left(\alpha_{k_{0}}^{(0)}, \beta_{k_{0}}^{*}, \gamma_{k_{0}}^{*}\right)
\end{aligned}
$$

must hold as well. However, inequalities (104) - (106) imply that there is the

$$
\left\{\left\{\alpha_{i}^{*}, \beta_{i}^{*}, \gamma_{i}^{*}\right\}_{i \in\{\overline{1, N}\}\left\{k_{0}\right\}} \cup\left\{\alpha_{k_{0}}^{(0)}, \beta_{k_{0}}^{*}, \gamma_{k_{0}}^{*}\right\}\right\} \text {-stack equilibrium, }
$$

which is impossible. Supposing that $\left\{\alpha_{k_{0}}^{(0)}, \beta_{k_{0}}^{(0)}, \gamma_{k_{0}}^{*}\right\}$ is an equilibrium by $\alpha_{k_{0}}^{(0)} \neq \alpha_{k_{0}}^{*}$ and $\beta_{k_{0}}^{(0)} \neq \beta_{k_{0}}^{*}$ leads to inequalities

$$
F\left(\alpha_{k_{0}}, \beta_{k_{0}}^{(0)}, \gamma_{k_{0}}^{*}\right) \leqslant F\left(\alpha_{k_{0}}^{(0)}, \beta_{k_{0}}^{(0)}, \gamma_{k_{0}}^{*}\right) \quad \forall \alpha_{k_{0}} \in\left[a_{\min } ; a_{\max }\right]
$$


and

$$
G\left(\alpha_{k_{0}}^{(0)}, \beta_{k_{0}}, \gamma_{k_{0}}^{*}\right) \leqslant G\left(\alpha_{k_{0}}^{(0)}, \beta_{k_{0}}^{(0)}, \gamma_{k_{0}}^{*}\right) \quad \forall \beta_{k_{0}} \in\left[b_{\min } ; b_{\max }\right]
$$

and

$$
H\left(\alpha_{k_{0}}^{(0)}, \beta_{k_{0}}^{(0)}, \gamma_{k_{0}}\right) \leqslant H\left(\alpha_{k_{0}}^{(0)}, \beta_{k_{0}}^{(0)}, \gamma_{k_{0}}^{*}\right) \quad \forall \gamma_{k_{0}} \in\left[c_{\min } ; c_{\max }\right]
$$

Inequalities (107) - (109) imply that inequalities

$$
\begin{aligned}
& \sum_{k_{*} \in\{1, N\}\left\{\left\{k_{0}\right\}\right.} F\left(\alpha_{k_{*}}, \beta_{k_{*}}^{*}, \gamma_{k_{*}}^{*}\right)+F\left(\alpha_{k_{0}}, \beta_{k_{0}}^{(0)}, \gamma_{k_{0}}^{*}\right) \leqslant \\
\leqslant & \sum_{\left.k_{*} \in\{1, N\}\right\}\left\{k_{0}\right\}} F\left(\alpha_{k_{*}}^{*}, \beta_{k_{*}}^{*}, \gamma_{k_{*}}^{*}\right)+F\left(\alpha_{k_{0}}^{(0)}, \beta_{k_{0}}^{(0)}, \gamma_{k_{0}}^{*}\right)
\end{aligned}
$$

and

$$
\begin{aligned}
& \sum_{k_{*} \in\{1, N\}\left\{\left\{k_{0}\right\}\right.} G\left(\alpha_{k_{*}}^{*}, \beta_{k_{*}}, \gamma_{k_{*}}^{*}\right)+G\left(\alpha_{k_{0}}^{(0)}, \beta_{k_{0}}, \gamma_{k_{0}}^{*}\right) \leqslant \\
& \leqslant \sum_{k_{*} \in\{1, N\} \backslash\left\{k_{0}\right\}} G\left(\alpha_{k_{*}}^{*}, \beta_{k_{*}}^{*}, \gamma_{k_{*}}^{*}\right)+G\left(\alpha_{k_{0}}^{(0)}, \beta_{k_{0}}^{(0)}, \gamma_{k_{0}}^{*}\right)
\end{aligned}
$$

and

$$
\begin{aligned}
& \sum_{k_{*} \in\{1, N\}\left\{\left\{k_{0}\right\}\right.} H\left(\alpha_{k_{*}}^{*}, \beta_{k_{*}}^{*}, \gamma_{k_{*}}\right)+H\left(\alpha_{k_{0}}^{(0)}, \beta_{k_{0}}^{(0)}, \gamma_{k_{0}}^{*}\right) \leqslant \\
& \leqslant \sum_{k_{*} \in\{1, N\}\left\{k_{0}\right\}} H\left(\alpha_{k_{*}}^{*}, \beta_{k_{*}}^{*}, \gamma_{k_{*}}^{*}\right)+H\left(\alpha_{k_{0}}^{(0)}, \beta_{k_{0}}^{(0)}, \gamma_{k_{0}}^{*}\right)
\end{aligned}
$$

must hold as well. Then inequalities (110) - (112) imply that there is the

$$
\left\{\left\{\alpha_{i}^{*}, \beta_{i}^{*}, \gamma_{i}^{*}\right\}_{i \in\{\overline{1, N}\} \backslash\left\{k_{0}\right\}} \cup\left\{\alpha_{k_{0}}^{(0)}, \beta_{k_{0}}^{(0)}, \gamma_{k_{0}}^{*}\right\}\right\} \text {-stack equilibrium, }
$$

which is impossible again. The impossibility of other pure-strategy equilibrium cases in "short" games (27) is proved symmetrically.

In trimatrix games, which are a partial case of three-person games, the case when every "short" game has just a single pure-strategy equilibrium seems to be rarer than the case with multiple equilibria. This, however, does not diminish the importance of Theorem 1 along with Theorem 2 and Theorem 3. These assertions allow to build a simpler proof of a more generalized assertion.

Theorem 4. If each of $N$ games (27) by (16) - (26) and (29) - (38) has a nonempty set of equilibrium situations in pure strategies, and game (14) on product (15) by conditions (1) - (13) is equivalent to the succession of these games, then every pure-strategy equilibrium in game (14) is a stack of any 
respective $N$ equilibria in games (27). Apart from the stack, there are no other pure-strategy equilibria in game (14).

Proof. Let the $i$-th game have $J_{i}$ equilibria

$$
\left\{\alpha_{i j_{i}}^{*}, \beta_{i j_{i}}^{*}, \gamma_{i j_{i}}^{*}\right\}_{j_{i}=1}^{J_{i}}
$$

by $J_{i} \in \bullet$, where

$$
\alpha_{i j_{i}}^{*} \in\left[a_{\min } ; a_{\max }\right], \beta_{i j_{i}}^{*} \in\left[b_{\min } ; b_{\max }\right], \gamma_{i j_{i}}^{*} \in\left[c_{\min } ; c_{\max }\right]
$$

Then

$$
F\left(\alpha_{i}, \beta_{i j_{i}}^{*}, \gamma_{i j_{i}}^{*}\right) \leqslant F\left(\alpha_{i j_{i}}^{*}, \beta_{i j_{i}}^{*}, \gamma_{i j_{i}}^{*}\right) \quad \forall \alpha_{i} \in\left[a_{\min } ; a_{\max }\right]
$$

and

$$
G\left(\alpha_{i j_{i}}^{*}, \beta_{i}, \gamma_{i j_{i}}^{*}\right) \leqslant G\left(\alpha_{i j_{i}}^{*}, \beta_{i j_{i}}^{*}, \gamma_{i j_{i}}^{*}\right) \quad \forall \beta_{i} \in\left[b_{\min } ; b_{\max }\right]
$$

and

$$
H\left(\alpha_{i j_{i}}^{*}, \beta_{i j_{i}}^{*}, \gamma_{i}\right) \leqslant H\left(\alpha_{i j_{i}}^{*}, \beta_{i j_{i}}^{*}, \gamma_{i j_{i}}^{*}\right) \quad \forall \gamma_{i} \in\left[c_{\min } ; c_{\max }\right]
$$

whence

$$
\sum_{i=1}^{N} F\left(\alpha_{i}, \beta_{i j_{i}}^{*}, \gamma_{i j_{i}}^{*}\right) \leqslant \sum_{i=1}^{N} F\left(\alpha_{i j_{i}}^{*}, \beta_{i j_{i}}^{*}, \gamma_{i j_{i}}^{*}\right)
$$

and

$$
\sum_{i=1}^{N} G\left(\alpha_{i j_{i}}^{*}, \beta_{i}, \gamma_{i j_{i}}^{*}\right) \leqslant \sum_{i=1}^{N} G\left(\alpha_{i j_{i}}^{*}, \beta_{i j_{i}}^{*}, \gamma_{i j_{i}}^{*}\right)
$$

and

$$
\sum_{i=1}^{N} H\left(\alpha_{i j_{i}}^{*}, \beta_{i j_{i}}^{*}, \gamma_{i}\right) \leqslant \sum_{i=1}^{N} H\left(\alpha_{i j_{i}}^{*}, \beta_{i j_{i}}^{*}, \gamma_{i j_{i}}^{*}\right)
$$

Inequalities (116) - (118) directly imply the

$$
\left\{\alpha_{i j_{i}}^{*}, \beta_{i j_{i}}^{*}, \gamma_{i j_{i}}^{*}\right\}_{i=1}^{N} \text {-stack equilibrium }
$$

for every $j_{i} \in\left\{\overline{1, J_{i}}\right\}$ by $i=\overline{1, N}$. Apart from stacks (119), there are no other pure-strategy equilibria in game (14) owing to Theorem 3 along with Theorem 2.

It is quite obvious that Theorems $1-4$ are valid for any three-person games whose players are constrained (forced) to use staircase-function strategies, i. e., they are valid for trimatrix games (with staircase-function strategies) as well. It remains only to study a possibility of equilibria in mixed strategies in such trimatrix games. 


\section{Representation by a succession of trimatrix games}

Along with discrete time intervals, players may be forced to act within a finite subset of possible values of their pure strategies. That is, these values are

$$
a_{\min }=a^{(0)}<a^{(1)}<a^{(2)}<\ldots<a^{(M-1)}<a^{(M)}=a_{\max }
$$

and

$$
b_{\min }=b^{(0)}<b^{(1)}<b^{(2)}<\ldots<b^{(Q-1)}<b^{(Q)}=b_{\max }
$$

and

$$
c_{\min }=c^{(0)}<c^{(1)}<c^{(2)}<\ldots<c^{(S-1)}<c^{(S)}=c_{\max }
$$

for the first and second and third players, respectively $(M \in \mathbb{N}$ and $Q \in \mathbb{N}$ and $S \in \mathbb{N}$ ). Then the succession of $N$ continuous games (27) by (16) - (26) and (29) - (38) becomes a succession of $N$ trimatrix games

$$
\left\langle\left\{\left\{a^{(m-1)}\right\}_{m=1}^{M+1},\left\{b^{(q-1)}\right\}_{q=1}^{Q+1},\left\{c^{(s-1)}\right\}_{s=1}^{S+1}\right\},\left\{\mathbf{F}_{i}, \mathbf{G}_{i}, \mathbf{H}_{i}\right\}\right\rangle
$$

with first player's payoff matrices

$$
\mathbf{F}_{i}=\left[\varphi_{i m q s}\right]_{(M+1) \times(Q+1) \times(S+1)}
$$

whose elements are

$$
\varphi_{\text {imqs }}=\int_{\left[\tau^{(i-1)} ; \tau^{(i)}\right)} f\left(a^{(m-1)}, b^{(q-1)}, c^{(s-1)}, t\right) d \mu(t) \text { for } i=\overline{1, N-1}
$$

and

$$
\varphi_{\text {Nmqs }}=\int_{\left[\tau^{(N-1)} ; \tau^{(N)}\right]} f\left(a^{(m-1)}, b^{(q-1)}, c^{(s-1)}, t\right) d \mu(t),
$$

with second player's payoff matrices

$$
\mathbf{G}_{i}=\left[\rho_{\text {imqs }}\right]_{(M+1) \times(Q+1) \times(S+1)}
$$

whose elements are

$$
\rho_{\text {imqs }}=\int_{\left[\tau^{(i-1)} ; \tau^{(i)}\right)} g\left(a^{(m-1)}, b^{(q-1)}, c^{(s-1)}, t\right) d \mu(t) \text { for } i=\overline{1, N-1}
$$

and

$$
\rho_{N m q s}=\int_{\left[\tau^{(N-1)} ; \tau^{(N)}\right]} g\left(a^{(m-1)}, b^{(q-1)}, c^{(s-1)}, t\right) d \mu(t),
$$


and with third player's payoff matrices

$$
\mathbf{H}_{i}=\left[\theta_{\text {imqs }}\right]_{(M+1) \times(Q+1) \times(S+1)}
$$

whose elements are

$$
\theta_{\text {imqs }}=\int_{\left[\tau^{(i-1)} ; \tau^{(i)}\right)} h\left(a^{(m-1)}, b^{(q-1)}, c^{(s-1)}, t\right) d \mu(t) \text { for } i=\overline{1, N-1}
$$

and

$$
\theta_{\text {Nmqs }}=\int_{\left[\tau^{(N-1)} ; \tau^{(N)}\right]} h\left(a^{(m-1)}, b^{(q-1)}, c^{(s-1)}, t\right) d \mu(t) .
$$

It is well-known that a finite three-person game always has an equilibrium either in pure or mixed strategies. So, if game (14) is made equivalent to a series of trimatrix games (or, in other words, is represented by a succession of trimatrix games), then it is easy to see that, unlike the representation with continuous games (27) by (16) - (26) and (29) - (38), the game always has a solution (at least, in mixed strategies).

Theorem 5. If game (14) on product (15) by conditions (1) - (13) is equivalent to the succession of $N$ trimatrix games (123) by (124) - (129), then the game is always solved as a stack of respective equilibria in these trimatrix games. Apart from the stack, there are no other equilibria in game (14).

Proof. An equilibrium situation in the trimatrix game always exists, either in pure or mixed strategies. Denote by

$$
\mathbf{U}_{i}=\left[u_{i}^{(m)}\right]_{1 \times(M+1)}
$$

and

$$
\mathbf{Z}_{i}=\left[z_{i}^{(q)}\right]_{1 \times(Q+1)}
$$

and

$$
\mathbf{W}_{i}=\left[w_{i}^{(s)}\right]_{1 \times(S+1)}
$$

the mixed strategies of the first and second and third players, respectively, in trimatrix game (123). The respective sets of mixed strategies of the first and second and third players are

$$
\boldsymbol{U}=\left\{\mathbf{U}_{i} \in \mathbb{R}^{M+1}: u_{i}^{(m)} \geqslant 0, \sum_{m=1}^{M+1} u_{i}^{(m)}=1\right\}
$$

and

$$
\boldsymbol{Z}=\left\{\mathbf{Z}_{i} \in \mathbb{R}^{Q+1}: z_{i}^{(q)} \geqslant 0, \sum_{q=1}^{Q+1} z_{i}^{(q)}=1\right\}
$$


and

$$
\boldsymbol{W}=\left\{\mathbf{W}_{i} \in \mathbb{R}^{S+1}: w_{i}^{(s)} \geqslant 0, \sum_{s=1}^{S+1} w_{i}^{(s)}=1\right\},
$$

so $\mathbf{U}_{i} \in \boldsymbol{U}, \quad \mathbf{Z}_{i} \in \boldsymbol{Z}, \quad \mathbf{W}_{i} \in \boldsymbol{W}$, and $\left\{\mathbf{U}_{i}, \mathbf{Z}_{i}, \mathbf{W}_{i}\right\}$ is a situation in game (123), where $J_{i}$ equilibria exist, $J_{i} \in \bullet$. Let $\left\{\mathbf{U}_{i j_{i}}^{*}, \mathbf{Z}_{i j_{i}}^{*}, \mathbf{W}_{i j_{i}}^{*}\right\}_{i=1}^{N}$ be equilibria in $N$ games (123) by (124) - (129), where

$$
\mathbf{U}_{i j_{i}}^{*}=\left[u_{i j_{i}}^{(m)^{*}}\right]_{1 \times(M+1)} \in \boldsymbol{U}
$$

and

$$
\mathbf{Z}_{i j_{i}}^{*}=\left[z_{i j_{i}}^{(q)^{*}}\right]_{1 \times(Q+1)} \in \boldsymbol{Z}
$$

and

$$
\mathbf{W}_{i j_{i}}^{*}=\left[w_{i j_{i}}^{(s)^{*}}\right]_{1 \times(S+1)} \in \boldsymbol{W} .
$$

Henceforward, the proof is similar to that in Theorem 4. For equilibria $\left\{\mathbf{U}_{i j_{i}}^{*}, \mathbf{Z}_{i j_{i}}^{*}, \mathbf{W}_{i j_{i}}^{*}\right\}_{i=1}^{N}$ by (133) - (135), inequalities

$$
\begin{aligned}
& \sum_{m=1}^{M+1} \sum_{q=1}^{Q+1} \sum_{s=1}^{S+1} \varphi_{i m q s} u_{i}^{(m)} z_{i j_{i}}^{(q)^{*}} w_{i j_{i}}^{(s)^{*}}= \\
& =\sum_{m=1}^{M+1} \sum_{q=1}^{Q+1} \sum_{s=1}^{S+1} u_{i}^{(m)} z_{i j_{i}}^{(q)^{*}} w_{i j_{i}}^{(s)^{*}} \int_{\left[\tau^{(i-1)} ; \tau^{(i)}\right)} f\left(a^{(m-1)}, b^{(q-1)}, c^{(s-1)}, t\right) d \mu(t) \leqslant \\
& \leqslant \sum_{m=1}^{M+1} \sum_{q=1}^{Q+1} \sum_{s=1}^{S+1} u_{i j_{i}}^{(m)^{*}} z_{i j_{i}}^{(q)^{*}} w_{i j_{i}}^{(s)^{*}} \int_{\left[\tau^{(i-1)} ; \tau^{(i)}\right)} f\left(a^{(m-1)}, b^{(q-1)}, c^{(s-1)}, t\right) d \mu(t)= \\
& =\sum_{m=1}^{M+1} \sum_{q=1}^{Q+1} \sum_{s=1}^{S+1} \varphi_{i m q s} u_{i j_{i}}^{(m)^{*}} z_{i j_{i}}^{(q)^{*}} w_{i j_{i}}^{(s)^{*}} \quad \forall \mathbf{U}_{i}=\left[u_{i}^{(m)}\right]_{1 \times(M+1)} \in \boldsymbol{U} \text { for } i=\overline{1, N-1} \text {, } \\
& \sum_{m=1}^{M+1} \sum_{q=1}^{Q+1} \sum_{s=1}^{S+1} \varphi_{N m q s} u_{N}^{(m)} z_{N j_{N}}^{(q)^{*}} w_{N j_{N}}^{(s)^{*}}= \\
& =\sum_{m=1}^{M+1} \sum_{q=1}^{Q+1} \sum_{s=1}^{S+1} u_{N}^{(m)} z_{N j_{N}}^{(q)^{*}} w_{N j_{N}}^{(s)^{*}} \int_{\left[\tau^{(N-1)} ; \tau^{(N)}\right]} f\left(a^{(m-1)}, b^{(q-1)}, c^{(s-1)}, t\right) d \mu(t) \leqslant \\
& \leqslant \sum_{m=1}^{M+1} \sum_{q=1}^{Q+1} \sum_{s=1}^{S+1} u_{N j_{N}}^{(m)^{*}} z_{N j_{N}}^{(q) *} w_{N j_{N}}^{(s)} \int_{\left[\tau^{(N-1)} ; \tau^{(N)}\right]} f\left(a^{(m-1)}, b^{(q-1)}, c^{(s-1)}, t\right) d \mu(t)=
\end{aligned}
$$




$$
=\sum_{m=1}^{M+1} \sum_{q=1}^{Q+1} \sum_{s=1}^{S+1} \varphi_{N m q s} u_{N j_{N}}^{(m)^{*}} z_{N j_{N}}^{(q)^{*}} w_{N j_{N}}^{(s)^{*}} \quad \forall \mathbf{U}_{N}=\left[u_{N}^{(m)}\right]_{1 \times(M+1)} \in \boldsymbol{U}
$$

and inequalities

$$
\begin{aligned}
& \sum_{m=1}^{M+1} \sum_{q=1}^{Q+1} \sum_{s=1}^{S+1} \rho_{i m q s} u_{i j_{i}}^{(m)^{*}} z_{i}^{(q)} w_{i j_{i}}^{(s)^{*}}= \\
& =\sum_{m=1}^{M+1} \sum_{q=1}^{Q+1} \sum_{s=1}^{S+1} u_{i j_{i}}^{(m)^{*}} z_{i}^{(q)} w_{i j_{i}}^{(s)^{*}} \int_{\left[\tau^{(i-1)} ; \tau^{(i)}\right)} g\left(a^{(m-1)}, b^{(q-1)}, c^{(s-1)}, t\right) d \mu(t) \leqslant \\
& \leqslant \sum_{m=1}^{M+1} \sum_{q=1}^{Q+1} \sum_{s=1}^{S+1} u_{i j_{i}}^{(m)^{*}} z_{i j_{i}}^{(q)^{*}} w_{i j_{i}}^{(s)^{*}} \int_{\left[\tau^{(i-1)} ; \tau^{(i)}\right)} g\left(a^{(m-1)}, b^{(q-1)}, c^{(s-1)}, t\right) d \mu(t)= \\
& =\sum_{m=1}^{M+1} \sum_{q=1}^{Q+1} \sum_{s=1}^{S+1} \rho_{i m q s} u_{i j_{i}}^{(m)^{*}} z_{i j_{i}}^{(q)^{*}} w_{i j_{i}}^{(s)^{*}} \quad \forall \mathbf{Z}_{i}=\left[z_{i}^{(q)}\right]_{1 \times(Q+1)} \in \boldsymbol{Z} \text { for } i=\overline{1, N-1} \text {, } \\
& \sum_{m=1}^{M+1} \sum_{q=1}^{Q+1} \sum_{s=1}^{S+1} \rho_{N m q s} u_{N j_{N}}^{(m)^{*}} z_{N}^{(q)} w_{N j_{N}}^{(s)^{*}}= \\
& =\sum_{m=1}^{M+1} \sum_{q=1}^{Q+1} \sum_{s=1}^{S+1} u_{N j_{N}}^{(m)^{*}} z_{N}^{(q)} w_{N j_{N}}^{(s)^{*}} \int_{\left[\tau^{(N-1)} ; \tau^{(N)}\right]} g\left(a^{(m-1)}, b^{(q-1)}, c^{(s-1)}, t\right) d \mu(t) \leqslant \\
& \leqslant \sum_{m=1}^{M+1} \sum_{q=1}^{Q+1} \sum_{s=1}^{S+1} u_{N j_{N}}^{(m)^{*}} z_{N j_{N}}^{(q)^{*}} w_{N j_{N}}^{(s)^{*}} \int_{\left[\tau^{(N-1)} ; \tau^{(N)}\right]} g\left(a^{(m-1)}, b^{(q-1)}, c^{(s-1)}, t\right) d \mu(t)= \\
& =\sum_{m=1}^{M+1} \sum_{q=1}^{Q+1} \sum_{s=1}^{S+1} \rho_{N m q s} u_{N j_{N}}^{(m)^{*}} z_{N j_{N}}^{(q)^{*}} w_{N j_{N}}^{(s)} \quad \forall \mathbf{Z}_{N}=\left[z_{N}^{(q)}\right]_{1 \times(Q+1)} \in \boldsymbol{Z},
\end{aligned}
$$

and inequalities

$$
\begin{gathered}
\sum_{m=1}^{M+1} \sum_{q=1}^{Q+1} \sum_{s=1}^{S+1} \theta_{i m q s} u_{i j_{i}}^{(m)^{*}} z_{i j_{i}}^{(q)^{*}} w_{i}^{(s)}= \\
=\sum_{m=1}^{M+1} \sum_{q=1}^{Q+1} \sum_{s=1}^{S+1} u_{i j_{i}}^{(m)^{*}} z_{i j_{i}}^{(q)^{*}} w_{i}^{(s)} \int_{\left[\tau^{(i-1)} ; \tau^{(i)}\right)} h\left(a^{(m-1)}, b^{(q-1)}, c^{(s-1)}, t\right) d \mu(t) \leqslant \\
\leqslant \sum_{m=1}^{M+1} \sum_{q=1}^{Q+1} \sum_{s=1}^{S+1} u_{i j_{i}}^{(m)^{*}} z_{i j_{i}}^{(q)^{*}} w_{i j_{i}}^{(s)^{*}} \int_{\left[\tau^{(i-1)} ; \tau^{(i)}\right)} h\left(a^{(m-1)}, b^{(q-1)}, c^{(s-1)}, t\right) d \mu(t)= \\
=\sum_{m=1}^{M+1} \sum_{q=1}^{Q+1} \sum_{s=1}^{S+1} \theta_{i m q s} u_{i j_{i}}^{(m)^{*}} z_{i j_{i}}^{(q)^{*}} w_{i j_{i}}^{(s)^{*}} \quad \forall \mathbf{W}_{i}=\left[w_{i}^{(s)}\right]_{1 \times(S+1)} \in \boldsymbol{W} \text { for } i=\overline{1, N-1,}
\end{gathered}
$$




$$
\begin{gathered}
\sum_{m=1}^{M+1} \sum_{q=1}^{Q+1} \sum_{s=1}^{S+1} \theta_{N m q s} u_{N j_{N}}^{(m)^{*}} z_{N j_{N}}^{(q) *} w_{N}^{(s)}= \\
=\sum_{m=1}^{M+1} \sum_{q=1}^{Q+1} \sum_{s=1}^{S+1} u_{N j_{N}}^{(m)^{*}} z_{N j_{N}}^{(q) *} w_{N}^{(s)} \int_{\left[\tau^{(N-1)} ; \tau^{(N)}\right]} h\left(a^{(m-1)}, b^{(q-1)}, c^{(s-1)}, t\right) d \mu(t) \leqslant \\
\leqslant \sum_{m=1}^{M+1} \sum_{q=1}^{Q+1} \sum_{s=1}^{S+1} u_{N j_{N}}^{(m)^{*}} z_{N j_{N}}^{(q)^{*}} w_{N j_{N}}^{(s)^{*}} \int_{\left[\tau^{(N-1)} ; \tau^{(N)}\right]} h\left(a^{(m-1)}, b^{(q-1)}, c^{(s-1)}, t\right) d \mu(t)= \\
=\sum_{m=1}^{M+1} \sum_{q=1}^{Q+1} \sum_{s=1}^{S+1} \theta_{N m q s} u_{N j_{N}}^{(m)^{*}} z_{N j_{N}}^{(q) *} w_{N j_{N}}^{(s)^{*}} \quad \forall \mathbf{W}_{N}=\left[w_{N}^{(s)}\right]_{1 \times(S+1)} \in \boldsymbol{W}
\end{gathered}
$$

hold. So, inequalities

$$
\begin{aligned}
& \sum_{i=1}^{N-1} \sum_{m=1}^{M+1} \sum_{q=1}^{Q+1} \sum_{s=1}^{S+1} \varphi_{i m q s} u_{i}^{(m)} z_{i j_{i}}^{(q)^{*}} w_{i j_{i}}^{(s)^{*}}+ \\
& +\sum_{m=1}^{M+1} \sum_{q=1}^{Q+1} \sum_{s=1}^{S+1} \varphi_{N m q s} u_{N}^{(m)} z_{N j_{N}}^{(q)^{*}} w_{N j_{N}}^{(s)^{*}}= \\
& =\sum_{i=1}^{N-1}\left(\sum_{m=1}^{M+1} \sum_{q=1}^{Q+1} \sum_{s=1}^{S+1} u_{i}^{(m)} z_{i j_{i}}^{(q)^{*}} w_{i j_{i}}^{(s)^{*}} \int_{\left[\tau^{(i-1)} ; \tau^{(i)}\right)} f\left(a^{(m-1)}, b^{(q-1)}, c^{(s-1)}, t\right) d \mu(t)\right)+ \\
& +\sum_{m=1}^{M+1} \sum_{q=1}^{Q+1} \sum_{s=1}^{S+1} u_{N}^{(m)} z_{N j_{N}}^{(q)^{*}} w_{N j_{N}}^{(s)^{*}} \int_{\left[\tau^{(N-1)} ; \tau^{(N)}\right]} f\left(a^{(m-1)}, b^{(q-1)}, c^{(s-1)}, t\right) d \mu(t) \leqslant \\
& \leqslant \sum_{i=1}^{N-1}\left(\sum_{m=1}^{M+1} \sum_{q=1}^{Q+1} \sum_{s=1}^{S+1} u_{i j_{i}}^{(m)^{*}} z_{i j_{i}}^{(q)^{*}} w_{i j_{i}}^{(s)^{*}} \int_{\left[\tau^{(i-1)} ; \tau^{(i)}\right)} f\left(a^{(m-1)}, b^{(q-1)}, c^{(s-1)}, t\right) d \mu(t)\right)+ \\
& +\sum_{m=1}^{M+1} \sum_{q=1}^{Q+1} \sum_{s=1}^{S+1} u_{N j_{N}}^{(m)^{*}} z_{N j_{N}}^{(q)^{*}} w_{N j_{N}}^{(s)^{*}} \int_{\left[\tau^{(N-1)} ; \tau^{(N)}\right]} f\left(a^{(m-1)}, b^{(q-1)}, c^{(s-1)}, t\right) d \mu(t)= \\
& =\sum_{i=1}^{N-1} \sum_{m=1}^{M+1} \sum_{q=1}^{Q+1} \sum_{s=1}^{S+1} \varphi_{i m q s} u_{i j_{i}}^{(m)^{*}} z_{i j_{i}}^{(q)^{*}} w_{i j_{i}}^{(s)^{*}}+ \\
& +\sum_{m=1}^{M+1} \sum_{q=1}^{Q+1} \sum_{s=1}^{S+1} \varphi_{N m q s} u_{N j_{N}}^{(m)^{*}} z_{N j_{N}}^{(q)^{*}} w_{N j_{N}}^{(s) *}
\end{aligned}
$$

and

$$
\sum_{i=1}^{N-1} \sum_{m=1}^{M+1} \sum_{q=1}^{Q+1} \sum_{s=1}^{S+1} \rho_{i m q s} u_{i j_{i}}^{(m)^{*}} z_{i}^{(q)} w_{i j_{i}}^{(s)^{*}}+
$$




$$
\begin{aligned}
& +\sum_{m=1}^{M+1} \sum_{q=1}^{Q+1} \sum_{s=1}^{S+1} \rho_{N m q s} u_{N j_{N}}^{(m)^{*}} z_{N}^{(q)} w_{N j_{N}}^{(s)^{*}}= \\
& =\sum_{i=1}^{N-1}\left(\sum_{m=1}^{M+1} \sum_{q=1}^{Q+1} \sum_{s=1}^{S+1} u_{i j_{i}}^{(m)^{*}} z_{i}^{(q)} w_{i j_{i}}^{(s)^{*}} \int_{\left[\tau^{(i-1)} ; \tau^{(i)}\right)} g\left(a^{(m-1)}, b^{(q-1)}, c^{(s-1)}, t\right) d \mu(t)\right)+ \\
& +\sum_{m=1}^{M+1} \sum_{q=1}^{Q+1} \sum_{s=1}^{S+1} u_{N j_{N}}^{(m)^{*}} z_{N}^{(q)} w_{N j_{N}}^{(s)^{*}} \int_{\left[\tau^{(N-1)} ; \tau^{(N)}\right]} g\left(a^{(m-1)}, b^{(q-1)}, c^{(s-1)}, t\right) d \mu(t) \leqslant \\
& \leqslant \sum_{i=1}^{N-1}\left(\sum_{m=1}^{M+1} \sum_{q=1}^{Q+1} \sum_{s=1}^{S+1} u_{i j_{i}}^{(m)^{*}} z_{i j_{i}}^{(q)^{*}} w_{i j_{i}}^{(s)^{*}} \int_{\left[\tau^{(i-1)} ; \tau^{(i)}\right)} g\left(a^{(m-1)}, b^{(q-1)}, c^{(s-1)}, t\right) d \mu(t)\right)+ \\
& +\sum_{m=1}^{M+1} \sum_{q=1}^{Q+1} \sum_{s=1}^{S+1} u_{N j_{N}}^{(m)^{*}} z_{N j_{N}}^{(q)^{*}} w_{N j_{N}}^{(s)^{*}} \int_{\left[\tau^{(N-1)} ; \tau^{(N)}\right]} g\left(a^{(m-1)}, b^{(q-1)}, c^{(s-1)}, t\right) d \mu(t)= \\
& =\sum_{i=1}^{N-1} \sum_{m=1}^{M+1} \sum_{q=1}^{Q+1} \sum_{s=1}^{S+1} \rho_{i m q s} u_{i j_{i}}^{(m)^{*}} z_{i j_{i}}^{(q)^{*}} w_{i j_{i}}^{(s)^{*}}+ \\
& +\sum_{m=1}^{M+1} \sum_{q=1}^{Q+1} \sum_{s=1}^{S+1} \rho_{N m q s} u_{N j_{N}}^{(m)} z_{N j_{N}}^{(q)^{*}} w_{N j_{N}}^{(s)^{*}}
\end{aligned}
$$

and

$$
\begin{aligned}
& \sum_{i=1}^{N-1} \sum_{m=1}^{M+1} \sum_{q=1}^{Q+1} \sum_{s=1}^{S+1} \theta_{i m q s} u_{i j_{i}}^{(m)^{*}} z_{i j_{i}}^{(q)^{*}} w_{i}^{(s)}+ \\
& +\sum_{m=1}^{M+1} \sum_{q=1}^{Q+1} \sum_{s=1}^{S+1} \theta_{N m q s} u_{N j_{N}}^{(m)^{*}} z_{N j_{N}}^{(q)^{*}} w_{N}^{(s)}= \\
= & \sum_{i=1}^{N-1}\left(\sum_{m=1}^{M+1} \sum_{q=1}^{Q+1} \sum_{s=1}^{S+1} u_{i j_{i}}^{(m)^{*}} z_{i j_{i}}^{(q)^{*}} w_{i}^{(s)} \int_{\left[\tau^{(i-1)} ; \tau^{(i)}\right)} h\left(a^{(m-1)}, b^{(q-1)}, c^{(s-1)}, t\right) d \mu(t)\right)+ \\
& +\sum_{m=1}^{M+1} \sum_{q=1}^{Q+1} \sum_{s=1}^{S+1} u_{N j_{N}}^{(m)} z_{N j_{N}}^{(q)^{*}} w_{N}^{(s)} \int_{\left[\tau^{(N-1)} ; \tau^{(N)}\right]} h\left(a^{(m-1)}, b^{(q-1)}, c^{(s-1)}, t\right) d \mu(t) \leqslant \\
\leqslant & \sum_{i=1}^{N-1}\left(\sum_{m=1}^{M+1} \sum_{q=1}^{Q+1} \sum_{s=1}^{S+1} u_{i j_{i}}^{(m)^{*}} z_{i j_{i}}^{(q)^{*}} w_{i j_{i}}^{(s)^{*}} \int_{\left[\tau^{(i-1)} ; \tau^{(i)}\right)} h\left(a^{(m-1)}, b^{(q-1)}, c^{(s-1)}, t\right) d \mu(t)\right)+ \\
& +\sum_{m=1}^{M+1} \sum_{q=1}^{Q+1} \sum_{s=1}^{S+1} u_{N j_{N}}^{(m)^{*}} z_{N j_{N}}^{(q)^{*}} w_{N j_{N}}^{(s) *} \int_{\left[\tau^{(N-1)} ; \tau^{(N)}\right]} h\left(a^{(m-1)}, b^{(q-1)}, c^{(s-1)}, t\right) d \mu(t)=
\end{aligned}
$$




$$
\begin{aligned}
= & \sum_{i=1}^{N-1} \sum_{m=1}^{M+1} \sum_{q=1}^{Q+1} \sum_{s=1}^{S+1} \theta_{i m q s} u_{i j_{i}}^{(m)^{*}} z_{i j_{i}}^{(q)^{*}} w_{i j_{i}}^{(s)^{*}}+ \\
& +\sum_{m=1}^{M+1} \sum_{q=1}^{Q+1} \sum_{s=1}^{S+1} \theta_{N m q s} u_{N j_{N}}^{(m)^{*}} z_{N j_{N}}^{(q)^{*}} w_{N j_{N}}^{(s)^{*}}
\end{aligned}
$$

hold as well. Therefore, the stack of successive equilibria $\left\{\mathbf{U}_{i j_{i}}^{*}, \mathbf{Z}_{i j_{i}}^{*}, \mathbf{W}_{i j_{i}}^{*}\right\}_{i=1}^{N}$ is an equilibrium in game (14). The sub-assertion of that, apart from such stacks, there are no other equilibria in game (14) is proved similarly to Theorem 3 along with Theorem 2.

Clearly, inequalities $(113)-(115)$ by $i=\overline{1, N}$ are a partial case of inequalities (136) - (141). Inequalities (116) - (118) are a partial case of inequalities (142) - (144). In a way, Theorem 5 is a generalization of Theorem 4 for the case of finite game (14), which is correspondingly defined a product of staircase-function finite spaces. Nevertheless, stacking up pure-strategy equilibria and mixed-strategy equilibria of $(M+1) \times(Q+1) \times(S+1)$ trimatrix games (123) can be cumbersome. The best case is when every "short" game has a single pure-strategy equilibrium, although the likelihood of the best case is low.

The likeliest case is when those $N$ trimatrix games have multiple pure-strategy equilibria and mixed-strategy equilibria. To hit on a series of single-pure-strategyequilibrium trimatrix games, plainly speaking, many tries should be done. For instance, $5 \times 5 \times 5$ games, in which payoffs are generated by a $5 \times 5 \times 5$ standard-normally-distributed array multiplied by 10 and rounded to the nearest integers towards $-\infty$, have roughly $28 \%$ mixed-strategy equilibria only. Meanwhile, the percentage rate of the case when the game has one pure-strategy equilibrium is at least $37 \%$.

\section{Exemplification}

To exemplify how the suggested method solves trimatrix games defined on a product of staircase-function spaces (which are obviously finite), consider a case in which $t \in[1.2 \pi ; 2 \pi]$, the set of pure strategies of the first player is

$$
X=\{x(t), t \in[1.2 \pi ; 2 \pi]: 5 \leqslant x(t) \leqslant 6\} \subset \mathbb{L}_{2}[1.2 \pi ; 2 \pi],
$$

the set of pure strategies of the second player is

$$
Y=\{y(t), t \in[1.2 \pi ; 2 \pi]: 2 \leqslant y(t) \leqslant 4\} \subset \mathbb{L}_{2}[1.2 \pi ; 2 \pi],
$$

and the set of pure strategies of the third player is

$$
Z=\{z(t), t \in[1.2 \pi ; 2 \pi]: 1 \leqslant z(t) \leqslant 2.6\} \subset \mathbb{L}_{2}[1.2 \pi ; 2 \pi] .
$$

The players' payoff functionals (8) - (10) are 


$$
\begin{gathered}
F(x(t), y(t), z(t))=\int_{[1.2 \pi ; 2 \pi]} \sin (0.4 x y z t) d \mu(t), \\
G(x(t), y(t), z(t))=\int_{[1.2 \pi ; 2 \pi]} \cos (0.1 x y z t) d \mu(t), \\
H(x(t), y(t), z(t))=\int_{[1.2 \pi ; 2 \pi]} \sin \left(0.9 x y z t-\frac{\pi}{3}\right) d \mu(t) .
\end{gathered}
$$

The players are forced to use pure strategies $x(t)$ and $y(t)$ and $z(t)$ such that

$$
x(t) \in\{5+0.1 \cdot(m-1)\}_{m=1}^{11} \subset[5 ; 6]
$$

and

$$
y(t) \in\{2+0.5 \cdot(q-1)\}_{q=1}^{5} \subset[2 ; 4]
$$

and

$$
z(t) \in\{1+0.2 \cdot(s-1)\}_{s=1}^{9} \subset[1 ; 2.6],
$$

and they can change their values only at time points

$$
\left\{\tau^{(i)}\right\}_{i=1}^{7}=\{1.2 \pi+0.1 i \pi\}_{i=1}^{7} .
$$

Consequently, this game can be thought of as it is defined on parallelepiped lattice

$$
\begin{aligned}
\{5+0.1 \cdot(m-1)\}_{m=1}^{11} & \times\{2+0.5 \cdot(q-1)\}_{q=1}^{5} \times\{1+0.2 \cdot(s-1)\}_{s=1}^{9} \subset \\
& \subset[5 ; 6] \times[2 ; 4] \times[1 ; 2.6],
\end{aligned}
$$

that is this game is a succession of 8 finite $11 \times 5 \times 9$ (trimatrix) games

$$
\begin{gathered}
\left\langle\left\{\left\{a^{(m-1)}\right\}_{m=1}^{11},\left\{b^{(q-1)}\right\}_{q=1}^{5},\left\{c^{(s-1)}\right\}_{s=1}^{9}\right\},\left\{\mathbf{F}_{i}, \mathbf{G}_{i}, \mathbf{H}_{i}\right\}\right\rangle= \\
=\left\langle\left\{\{5+0.1 \cdot(m-1)\}_{m=1}^{11},\{2+0.5 \cdot(q-1)\}_{q=1}^{5},\{1+0.2 \cdot(s-1)\}_{s=1}^{9}\right\},\left\{\mathbf{F}_{i}, \mathbf{G}_{i}, \mathbf{H}_{i}\right\}\right\rangle
\end{gathered}
$$

with first player's payoff matrices $\left\{\mathbf{F}_{i}=\left[\varphi_{i m q s}\right]_{11 \times 5 \times 9}\right\}_{i=1}^{8}$ whose elements are

$$
\begin{gathered}
\varphi_{\text {imqs }}=\int_{[1.2 \pi+0.1 \cdot(i-1) \pi ; 1.2 \pi+0.1 i \pi)} f\left(a^{(m-1)}, b^{(q-1)}, c^{(s-1)}, t\right) d \mu(t)= \\
=\int_{[1.2 \pi+0.1 \cdot(i-1) \pi ; 1.2 \pi+0.1 i \pi)} f(5+0.1 \cdot(m-1), 2+0.5 \cdot(q-1), 1+0.2 \cdot(s-1), t) d \mu(t)= \\
=\int_{[1.2 \pi+0.1 \cdot(i-1) \pi ; 1.2 \pi+0.1 i \pi)} \sin (0.4 \cdot(5+0.1 \cdot(m-1))(2+0.5 \cdot(q-1))(1+0.2 \cdot(s-1)) t) d \mu(t)=
\end{gathered}
$$




$$
=\int_{[1.2 \pi+0.1 \cdot(i-1) \pi ; 1.2 \pi+0.1 i \pi)} \sin (0.004 t \cdot(49+m)(3+q)(4+s)) d \mu(t) \text { for } i=\overline{1,7}
$$

and

$$
\varphi_{8 m q s}=\int_{[1.9 \pi ; 2 \pi]} \sin (0.004 t \cdot(49+m)(3+q)(4+s)) d \mu(t),
$$

with second player's payoff matrices $\left\{\mathbf{G}_{i}=\left[\rho_{\text {imqs }}\right]_{11 \times 5 \times 9}\right\}_{i=1}^{8}$ whose elements are

$$
\begin{gathered}
\rho_{\text {imqs }}=\int_{[1.2 \pi+0.1 \cdot(i-1) \pi ; 1.2 \pi+0.1 i \pi)} g\left(a^{(m-1)}, b^{(q-1)}, c^{(s-1)}, t\right) d \mu(t)= \\
=\int_{[1.2 \pi+0.1 \cdot(i-1) \pi ; 1.2 \pi+0.1 i \pi)} g(5+0.1 \cdot(m-1), 2+0.5 \cdot(q-1), 1+0.2 \cdot(s-1), t) d \mu(t)= \\
=\int_{[1.2 \pi+0.1 \cdot(i-1) \pi ; 1.2 \pi+0.1 i \pi)} \cos (0.1 \cdot(5+0.1 \cdot(m-1))(2+0.5 \cdot(q-1))(1+0.2 \cdot(s-1)) t) d \mu(t)= \\
=\int_{[1.2 \pi+0.1 \cdot(i-1) \pi ; 1.2 \pi+0.1 i \pi)} \cos (0.001 t \cdot(49+m)(3+q)(4+s)) d \mu(t) \text { for } i=\overline{1,7}
\end{gathered}
$$

and

$$
\rho_{8 m q s}=\int_{[1.9 \pi ; 2 \pi]} \cos (0.001 t \cdot(49+m)(3+q)(4+s)) d \mu(t),
$$

and with third player's payoff matrices $\left\{\mathbf{H}_{i}=\left[\theta_{\text {imqs }}\right]_{11 \times 5 \times 9}\right\}_{i=1}^{8}$ whose elements are

$$
\begin{gathered}
\theta_{\text {imqs }}=\int_{[1.2 \pi+0.1 \cdot(i-1) \pi ; 1.2 \pi+0.1 i \pi)} h\left(a^{(m-1)}, b^{(q-1)}, c^{(s-1)}, t\right) d \mu(t)= \\
=\int_{[1.2 \pi+0.1 \cdot(i-1) \pi ; 1.2 \pi+0.1 i \pi)} h(5+0.1 \cdot(m-1), 2+0.5 \cdot(q-1), 1+0.2 \cdot(s-1), t) d \mu(t)= \\
=\int_{[1.2 \pi+0.1 \cdot(i-1) \pi ; 1.2 \pi+0.1 i \pi)} \sin \left(0.9 \cdot(5+0.1 \cdot(m-1))(2+0.5 \cdot(q-1))(1+0.2 \cdot(s-1)) t-\frac{\pi}{3}\right) d \mu(t)= \\
=\int_{[1.2 \pi+0.1 \cdot(i-1) \pi ; 1.2 \pi+0.1 i \pi)} \sin \left(0.009 t \cdot(49+m)(3+q)(4+s)-\frac{\pi}{3}\right) d \mu(t) \text { for } i=\overline{1,7}(161)
\end{gathered}
$$

and

$$
\theta_{8 m q s}=\int_{[1.9 \pi ; 2 \pi]} \sin \left(0.009 t \cdot(49+m)(3+q)(4+s)-\frac{\pi}{3}\right) d \mu(t) .
$$


Each of the $11 \times 5 \times 9$ trimatrix games $(156)$ with $(157)-(162)$ is solved in pure strategies. Each of the games on intervals

$$
[1.5 \pi ; 1.6 \pi),[1.6 \pi ; 1.7 \pi),[1.7 \pi ; 1.8 \pi),[1.8 \pi ; 1.9 \pi)
$$

has a single pure-strategy equilibrium. The games on intervals

$$
[1.2 \pi ; 1.3 \pi),[1.3 \pi ; 1.4 \pi),[1.4 \pi ; 1.5 \pi),[1.9 \pi ; 2 \pi]
$$

have 2, 3, 2, and 2 pure-strategy equilibria, respectively. Consequently, there are

$$
2 \cdot 3 \cdot 2 \cdot 2=24
$$

equilibrium stacks. In other words, the three-person game by (145) - (154) has 24 pure strategy equilibria (Table 1). A half of them is presented in Figure 1, where each row contains an equilibrium pure strategy of the first (left), second (middle), and third players (right). The other half is presented in Figure 2. The respective players' payoffs

$$
\left\{F\left(\alpha_{i j_{i}}^{*}, \beta_{i j_{i}}^{*}, \gamma_{i j_{i}}^{*}\right), G\left(\alpha_{i j_{i}}^{*}, \beta_{i j_{i}}^{*}, \gamma_{i j_{i}}^{*}\right), H\left(\alpha_{i j_{i}}^{*}, \beta_{i j_{i}}^{*}, \gamma_{i j_{i}}^{*}\right)\right\}_{i=1}^{8}=\left\{\varphi_{i j_{i}}^{*}, \rho_{i j_{i}}^{*}, \theta_{i j_{i}}^{*}\right\}_{i=1}^{8}
$$

by

$$
j_{1} \in\{1,2\}, j_{2} \in\{1,2,3\}, j_{3} \in\{1,2\}, j_{4}=1, j_{5}=1, j_{6}=1, j_{7}=1, j_{8} \in\{1,2\}
$$

are presented in Table 2 . The 24 bar charts of payoff cumulative sums

$$
\left\{\sum_{k=1}^{i} \varphi_{k j_{k}}^{*}, \sum_{k=1}^{i} \rho_{k j_{k}}^{*}, \sum_{k=1}^{i} \theta_{k j_{k}}^{*}\right\}_{i=1}^{8}=\left\{\varphi_{\Sigma}^{(i)^{*}}, \rho_{\Sigma}^{(i)^{*}}, \theta_{\Sigma}^{(i)^{*}}\right\}_{i=1}^{8}
$$

by (163) are shown in Figure 3. The eventual payoffs of the players

$$
\left\{\sum_{k=1}^{8} \varphi_{k j_{k}}^{*}, \sum_{k=1}^{8} \rho_{k j_{k}}^{*}, \sum_{k=1}^{8} \theta_{k j_{k}}^{*}\right\}=\left\{\varphi_{\Sigma}^{(8)^{*}}, \rho_{\Sigma}^{(8)^{*}}, \theta_{\Sigma}^{(8)^{*}}\right\}
$$

vary with the stack (these payoffs are seen as the bars at $i=8$ in Figure 3). The first player's eventual payoff varies between 2.084618382 and 2.170187663 , which is $3.943 \%$ with respect to the maximum. The second player's eventual payoff varies between 2.1824161 and 2.361104 , which is $7.568 \%$ with respect to the maximum. The third player's eventual payoff varies considerably wider: it is between 0.908546892 and 1.146505582 , which is $20.755 \%$ with respect to the maximum.

After seeing those 24 stacks and payoffs (165), the arising question is quite obvious: which stack is the best? This question is the same as asked when equilibria are refined [4, $12,18,9]$. It is worth to note that the maximal eventual payoff for the second and third players is achieved at the 16-th (see the middle and right subplot of the fourth row in Figure 2) and 20-th (see the middle and right subplot of the eighth row in Figure 2) stacks, whereas the first player obtains its maximal payoff at the 10-th stack (see the left subplot of the third row from the bottom in Figure 1). The relative difference between the maximum (as it has been just mentioned above, it is 2.170187663 ) and the first player's payoff at the 16-th and 20-th stacks (which is 2.1328568 at both) is about $1.72 \%$. The aggregate payoff 
Table 1. The 24 pure strategy equilibria in the three-person game by (145) - (154)

\begin{tabular}{|c|c|c|c|c|c|c|c|c|c|c|c|c|c|c|c|c|c|c|}
\hline \multirow{2}{*}{ Interval } & \multicolumn{18}{|c|}{ Equilibrium stacks } \\
\hline & $x(t)$ & $y(t)$ & $z(t)$ & $x(t)$ & $y(t)$ & $z(t)$ & $x(t)$ & $y(t)$ & $z(t)$ & $x(t)$ & $y(t)$ & $z(t)$ & $x(t)$ & $y(t)$ & $z(t)$ & $x(t)$ & $y(t)$ & $z(t)$ \\
\hline$[1.2 \pi ; 1.3 \pi)$ & 5.7 & 3 & 1 & 5.7 & 3 & 1 & 5.7 & 3 & 1 & 5.7 & 3 & 1 & 5.7 & 3 & 1 & 5.7 & 3 & 1 \\
\hline$[1.3 \pi ; 1.4 \pi)$ & 5.2 & 2.5 & 1.2 & 5.2 & 2.5 & 1.2 & 5.2 & 2.5 & 1.2 & 5.2 & 2.5 & 1.2 & 5.2 & 3 & 1 & 5.2 & 3 & 1 \\
\hline$[1.4 \pi ; 1.5 \pi)$ & 5.2 & 2 & 1.4 & 5.2 & 2 & 1.4 & 6 & 2 & 1.2 & 6 & 2 & 1.2 & 5.2 & 2 & 1.4 & 5.2 & 2 & 1.4 \\
\hline$[1.5 \pi ; 1.6 \pi)$ & 5.7 & 2 & 1.2 & 5.7 & 2 & 1.2 & 5.7 & 2 & 1.2 & 5.7 & 2 & 1.2 & 5.7 & 2 & 1.2 & 5.7 & 2 & 1.2 \\
\hline$[1.6 \pi ; 1.7 \pi)$ & 5.1 & 2.5 & 1 & 5.1 & 2.5 & 1 & 5.1 & 2.5 & 1 & 5.1 & 2.5 & 1 & 5.1 & 2.5 & 1 & 5.1 & 2.5 & 1 \\
\hline$[1.7 \pi ; 1.8 \pi)$ & 6 & 2 & 1 & 6 & 2 & 1 & 6 & 2 & 1 & 6 & 2 & 1 & 6 & 2 & 1 & 6 & 2 & 1 \\
\hline$[1.8 \pi ; 1.9 \pi)$ & 5.7 & 2 & 1 & 5.7 & 2 & 1 & 5.7 & 2 & 1 & 5.7 & 2 & 1 & 5.7 & 2 & 1 & 5.7 & 2 & 1 \\
\hline$[1.9 \pi ; 2 \pi]$ & 5.2 & 2 & 1.8 & 5.4 & 2 & 1 & 5.2 & 2 & 1.8 & 5.4 & 2 & 1 & 5.2 & 2 & 1.8 & 5.4 & 2 & 1 \\
\hline$[1.2 \pi ; 1.3 \pi)$ & 5.7 & 3 & 1 & 5.7 & 3 & 1 & 5.7 & 3 & 1 & 5.7 & 3 & 1 & 5.7 & 3 & 1 & 5.7 & 3 & 1 \\
\hline$[1.3 \pi ; 1.4 \pi)$ & 5.2 & 3 & 1 & 5.2 & 3 & 1 & 5.6 & 2 & 1.4 & 5.6 & 2 & 1.4 & 5.6 & 2 & 1.4 & 5.6 & 2 & 1.4 \\
\hline$[1.4 \pi ; 1.5 \pi)$ & 6 & 2 & 1.2 & 6 & 2 & 1.2 & 5.2 & 2 & 1.4 & 5.2 & 2 & 1.4 & 6 & 2 & 1.2 & 6 & 2 & 1.2 \\
\hline$[1.5 \pi ; 1.6 \pi)$ & 5.7 & 2 & 1.2 & 5.7 & 2 & 1.2 & 5.7 & 2 & 1.2 & 5.7 & 2 & 1.2 & 5.7 & 2 & 1.2 & 5.7 & 2 & 1.2 \\
\hline$[1.6 \pi ; 1.7 \pi)$ & 5.1 & 2.5 & 1 & 5.1 & 2.5 & 1 & 5.1 & 2.5 & 1 & 5.1 & 2.5 & 1 & 5.1 & 2.5 & 1 & 5.1 & 2.5 & 1 \\
\hline$[1.7 \pi ; 1.8 \pi)$ & 6 & 2 & 1 & 6 & 2 & 1 & 6 & 2 & 1 & 6 & 2 & 1 & 6 & 2 & 1 & 6 & 2 & 1 \\
\hline$[1.8 \pi ; 1.9 \pi)$ & 5.7 & 2 & 1 & 5.7 & 2 & 1 & 5.7 & 2 & 1 & 5.7 & 2 & 1 & 5.7 & 2 & 1 & 5.7 & 2 & 1 \\
\hline$[1.9 \pi ; 2 \pi]$ & 5.2 & 2 & 1.8 & 5.4 & 2 & 1 & 5.2 & 2 & 1.8 & 5.4 & 2 & 1 & 5.2 & 2 & 1.8 & 5.4 & 2 & 1 \\
\hline$[1.2 \pi ; 1.3 \pi)$ & 6 & 2 & 1.4 & 6 & 2 & 1.4 & 6 & 2 & 1.4 & 6 & 2 & 1.4 & 6 & 2 & 1.4 & 6 & 2 & 1.4 \\
\hline$[1.3 \pi ; 1.4 \pi)$ & 5.2 & 2.5 & 1.2 & 5.2 & 2.5 & 1.2 & 5.2 & 2.5 & 1.2 & 5.2 & 2.5 & 1.2 & 5.2 & 3 & 1 & 5.2 & 3 & 1 \\
\hline$[1.4 \pi ; 1.5 \pi)$ & 5.2 & 2 & 1.4 & 5.2 & 2 & 1.4 & 6 & 2 & 1.2 & 6 & 2 & 1.2 & 5.2 & 2 & 1.4 & 5.2 & 2 & 1.4 \\
\hline$[1.5 \pi ; 1.6 \pi)$ & 5.7 & 2 & 1.2 & 5.7 & 2 & 1.2 & 5.7 & 2 & 1.2 & 5.7 & 2 & 1.2 & 5.7 & 2 & 1.2 & 5.7 & 2 & 1.2 \\
\hline$[1.6 \pi ; 1.7 \pi)$ & 5.1 & 2.5 & 1 & 5.1 & 2.5 & 1 & 5.1 & 2.5 & 1 & 5.1 & 2.5 & 1 & 5.1 & 2.5 & 1 & 5.1 & 2.5 & 1 \\
\hline$[1.7 \pi ; 1.8 \pi)$ & 6 & 2 & 1 & 6 & 2 & 1 & 6 & 2 & 1 & 6 & 2 & 1 & 6 & 2 & 1 & 6 & 2 & 1 \\
\hline$[1.8 \pi ; 1.9 \pi)$ & 5.7 & 2 & 1 & 5.7 & 2 & 1 & 5.7 & 2 & 1 & 5.7 & 2 & 1 & 5.7 & 2 & 1 & 5.7 & 2 & 1 \\
\hline$[1.9 \pi ; 2 \pi]$ & 5.2 & 2 & 1.8 & 5.4 & 2 & 1 & 5.2 & 2 & 1.8 & 5.4 & 2 & 1 & 5.2 & 2 & 1.8 & 5.4 & 2 & 1 \\
\hline$[1.2 \pi ; 1.3 \pi)$ & 6 & 2 & 1.4 & 6 & 2 & 1.4 & 6 & 2 & 1.4 & 6 & 2 & 1.4 & 6 & 2 & 1.4 & 6 & 2 & 1.4 \\
\hline$[1.3 \pi ; 1.4 \pi)$ & 5.2 & 3 & 1 & 5.2 & 3 & 1 & 5.6 & 2 & 1.4 & 5.6 & 2 & 1.4 & 5.6 & 2 & 1.4 & 5.6 & 2 & 1.4 \\
\hline$[1.4 \pi ; 1.5 \pi)$ & 6 & 2 & 1.2 & 6 & 2 & 1.2 & 5.2 & 2 & 1.4 & 5.2 & 2 & 1.4 & 6 & 2 & 1.2 & 6 & 2 & 1.2 \\
\hline$[1.5 \pi ; 1.6 \pi)$ & 5.7 & 2 & 1.2 & 5.7 & 2 & 1.2 & 5.7 & 2 & 1.2 & 5.7 & 2 & 1.2 & 5.7 & 2 & 1.2 & 5.7 & 2 & 1.2 \\
\hline$[1.6 \pi ; 1.7 \pi)$ & 5.1 & 2.5 & 1 & 5.1 & 2.5 & 1 & 5.1 & 2.5 & 1 & 5.1 & 2.5 & 1 & 5.1 & 2.5 & 1 & 5.1 & 2.5 & 1 \\
\hline$[1.7 \pi ; 1.8 \pi)$ & 6 & 2 & 1 & 6 & 2 & 1 & 6 & 2 & 1 & 6 & 2 & 1 & 6 & 2 & 1 & 6 & 2 & 1 \\
\hline$[1.8 \pi ; 1.9 \pi)$ & 5.7 & 2 & 1 & 5.7 & 2 & 1 & 5.7 & 2 & 1 & 5.7 & 2 & 1 & 5.7 & 2 & 1 & 5.7 & 2 & 1 \\
\hline$[1.9 \pi ; 2 \pi]$ & 5.2 & 2 & 1.8 & 5.4 & 2 & 1 & 5.2 & 2 & 1.8 & 5.4 & 2 & 1 & 5.2 & 2 & 1.8 & 5.4 & 2 & 1 \\
\hline
\end{tabular}




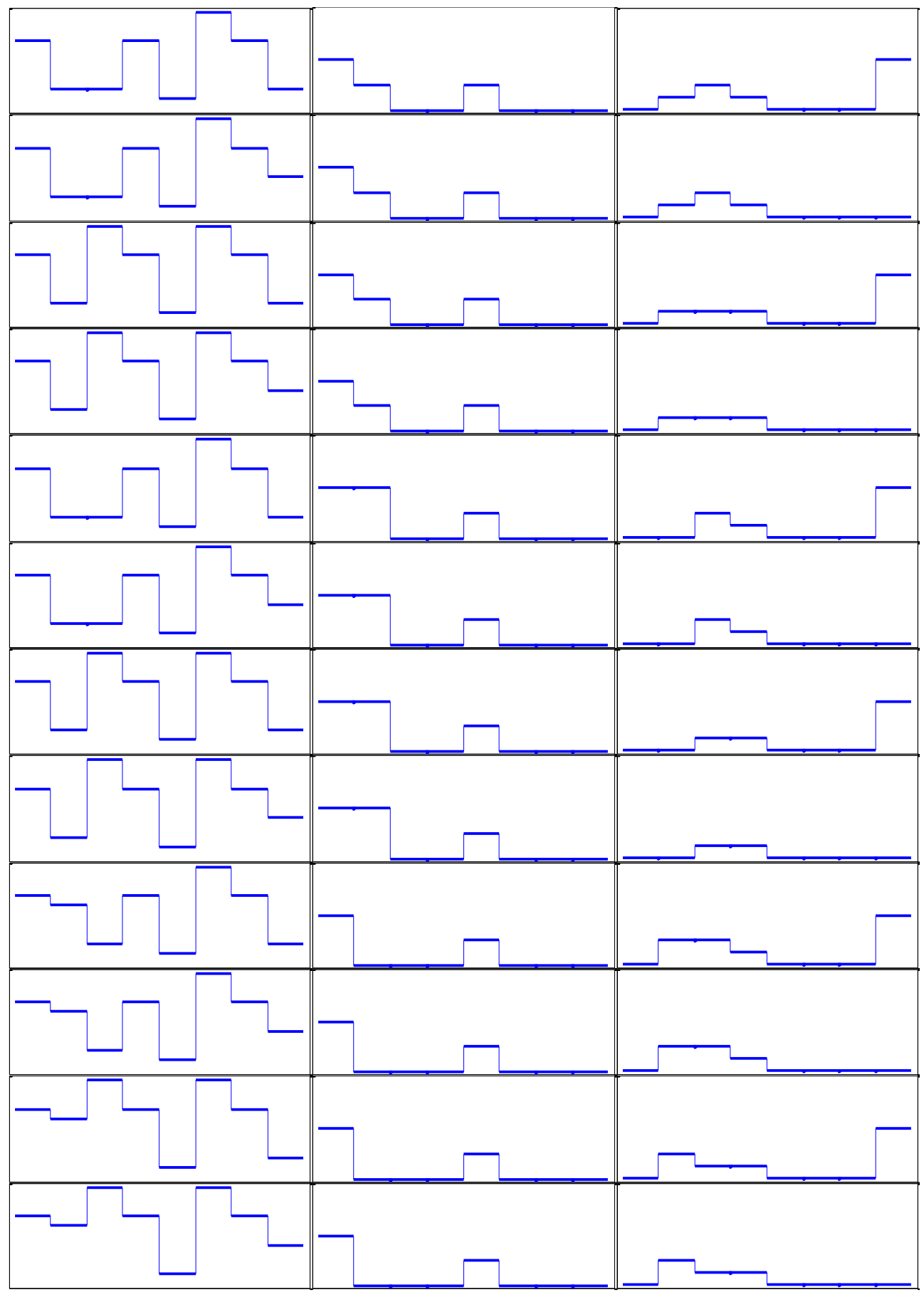

Figure 1. A half of the 24 pure-strategy equilibrium stacks in the three-person game by (145) - (154) 


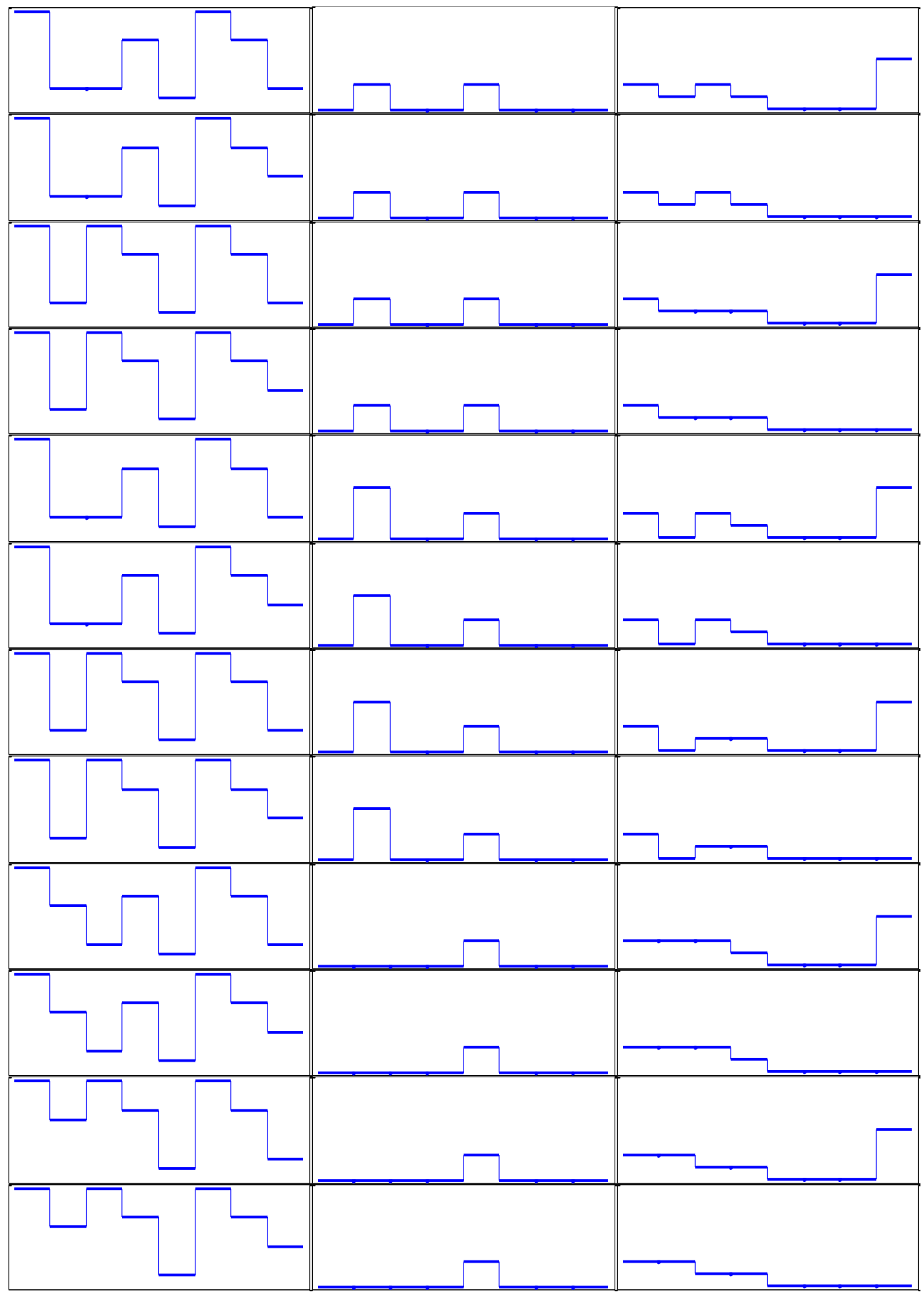

Figure 2. The other half of the 24 pure-strategy equilibrium stacks in the three-person game by (145) - (154) 
Table 2. The players' $(1,2,3)$ payoffs in the 24 pure strategy equilibria in the three-person game by (145) - (154)

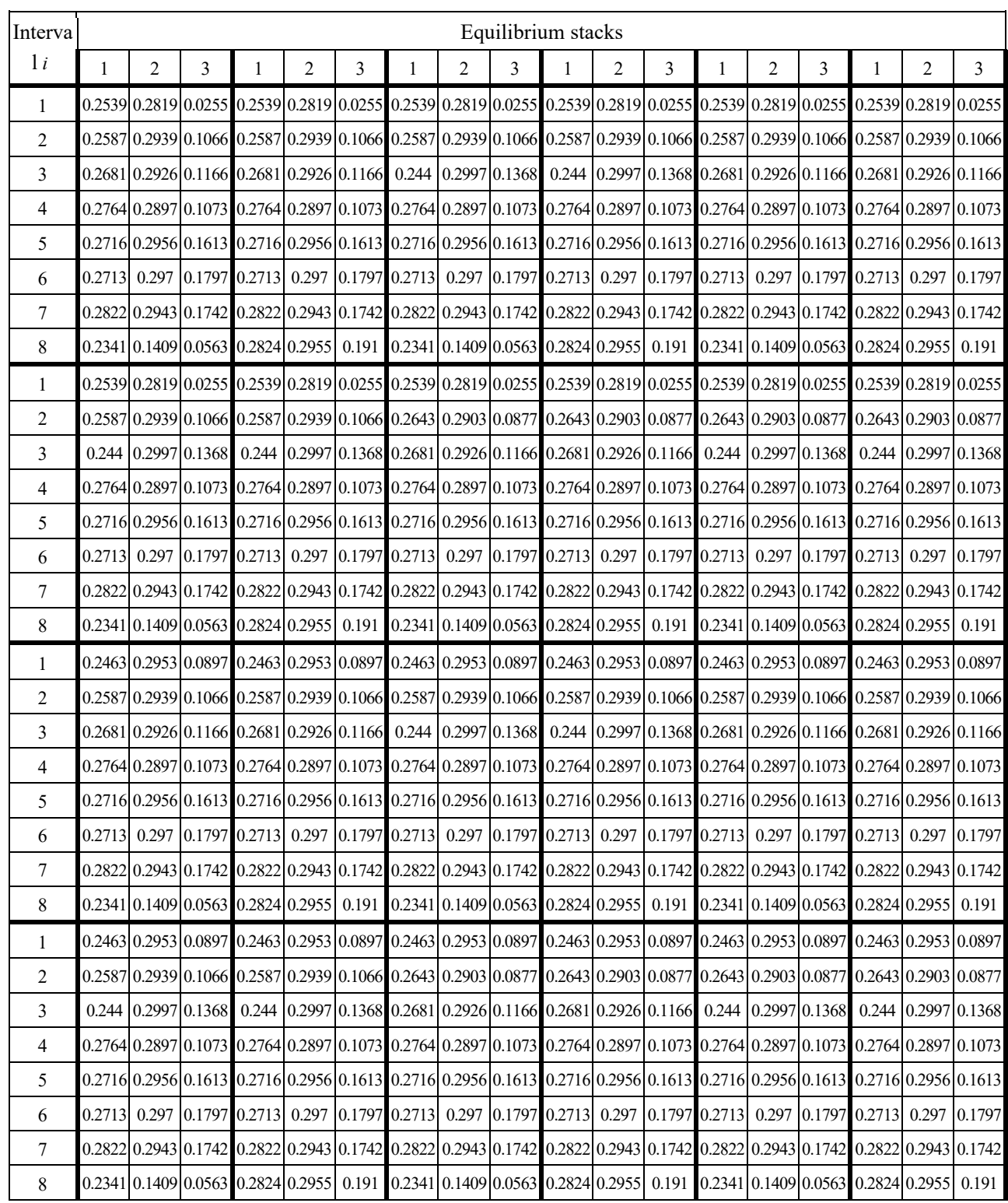

$$
\varphi_{\Sigma}^{(8)^{*}}+\rho_{\Sigma}^{(8)^{*}}+\theta_{\Sigma}^{(8)^{*}}
$$

achieves its maximum at the 16-th and 20-th stacks. Consequently, these equilibrium stacks are the best in the considered example. 

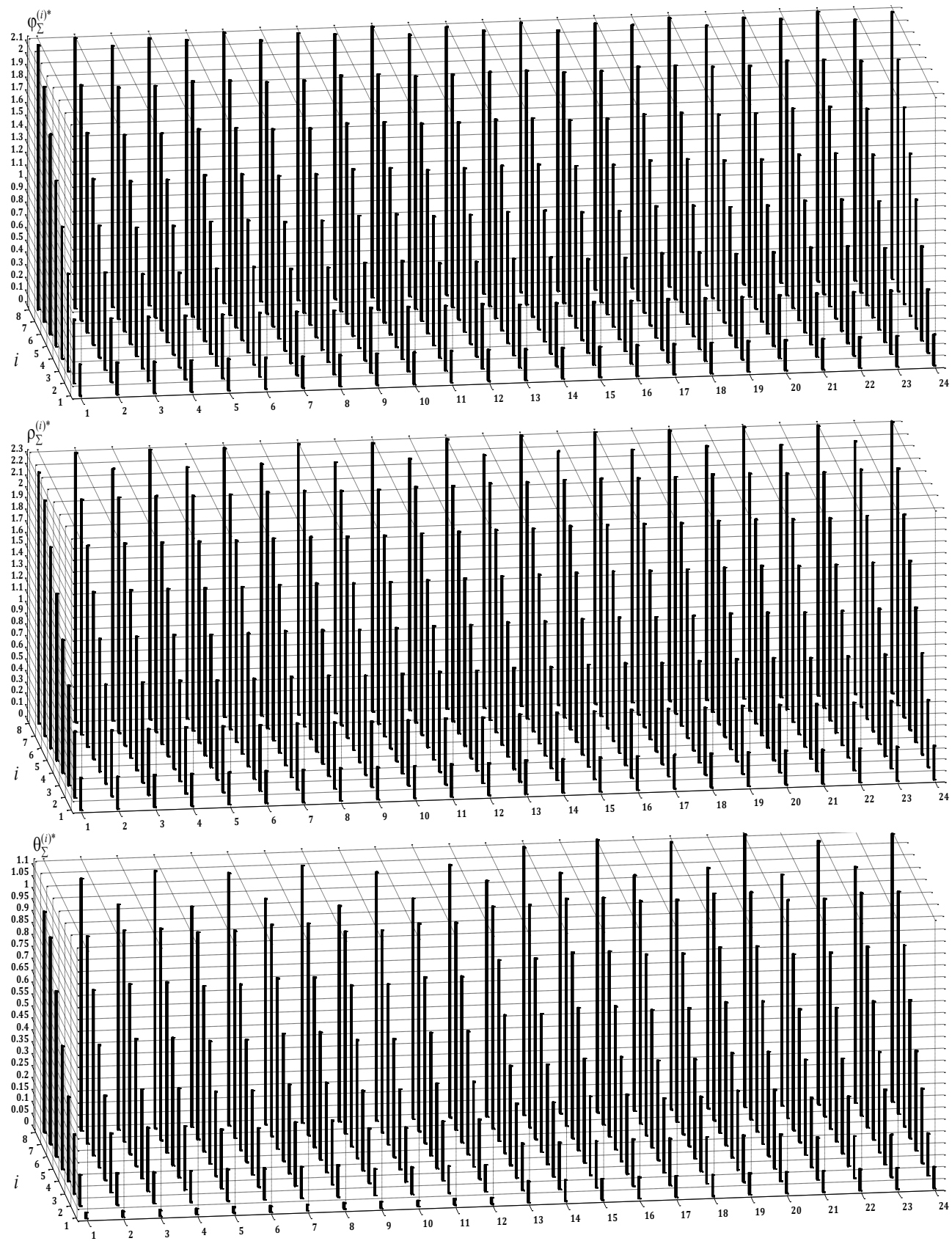

Figure 3. The 24 bar charts of payoff cumulative sums (164) for each player

Nevertheless, the example clearly shows that solving a succession of trimatrix games is far easier than tackling games whose players' pure strategies look like those staircase 
functions in Figure 1 and Figure 2. Even if not every trimatrix game has a single equilibrium, a solution of the initial game is built in the same way as (145) - (162). The only difference is that then there will be multiple stacked equilibria, which commonly induce instability of the players' behavior $[18,14,23,1,5,8]$. In particular, the considered example has shown how this instability problem can be solved. In general, the behavior instability is a serious problem in noncooperative games having multiple equilibria differing in the player's payoffs $[22,13,5,6]$. It is particularly solved by equilibria refinement with using domination efficiency along with maximin and the superoptimality rule $[12,18]$. The necessary condition is to have an asymmetry in the payoffs $[18,11,3]$. The asymmetry allows distinguish more profitable (and thus stable) equilibria, whereupon the best equilibrium (equilibria) or equilibrium stacks are selected. Otherwise, they are not distinguishable (like the 16-th and 20-th equilibrium stacks in the considered example).

The time spent on computation of a stack (any of those 24) is about 6 seconds. Surely, if time interval $[1.2 \pi ; 2 \pi]$ was broken into a set of narrower subintervals, the computation time would significantly increase. In general, the computation time has an exponential growth pattern as the size of the (cubical lattice) matrix increases.

\section{Discussion}

Continuous games are ever struggled to be approximated or rendered to finite games so that their solutions could be easily implemented and practiced [14, 18, 15, 21, 16]. However, even a finite (that is, trimatrix) game may be not tractable due to gigantic number of situations in the game. The presented method further "breaks" the initial ("long") game, defined on a product of staircase-function finite spaces, with a purpose to obtain an equilibrium in a more reasonable time. So, the method is far more tractable than a straightforward approach to solving directly the "long" trimatrix game would be.

Here, the tractability does not depend on the number of (time) intervals. Unless the sets of possible values of players' pure strategies are of order of hundreds or thousands (when searching for equilibria in a "short" trimatrix game may take a few seconds and more), the method is entirely applicable. Moreover, the presented method is a significant contribution to the mathematical game theory and practice for avoiding too complicated solution approaches resulting from game continuities and functional spaces of pure strategies. This is similar to preventing Einstellung effect in modeling $[16,7]$. The "breaking" of the initial game defined on a product of staircase-function finite spaces into a succession of "short" trimatrix games herein "deeinstellungizes" such noncooperative three-person games.

Stacking the "short" games' pure-strategy equilibria (by Theorem 4) is fulfilled trivially. When there is at least an equilibrium in mixed strategies for an interval (that actually falls within conditions of Theorem 5), the stacking is fulfilled as well implying that the resulting pure-mixed-strategy equilibrium in game (14) is realized successively, interval by interval, spending the same amount of time to implement both pure strategy and mixed strategy equilibria.

A drawback is that a "short" trimatrix game may be intractable itself if its size is too big. The size limitation depends on requirements from the administrator. If the interval breaking is over-thick, the "long" trimatrix game may be solved in an unreasonable amount 
of time (although every "short" game is tractable and solved relatively fast). After all, the size of the "short" trimatrix game should be made as small as possible.

\section{Conclusion}

Three-person games are used to model processes where three sides struggle for optimizing the distribution of the limited resources. Such processes are widespread in practice, where a strategy can be a complex set of simple actions ordinarily represented as a function of time. A three-person game defined on a product of staircase-function finite spaces is equivalent to a trimatrix game. Players' payoff matrices in this game are built very slowly, so it is impracticable to find any equilibria (as well as the other solution types) in such games using straightforwardly methods to solve a finite noncooperative game. However, the threeperson game is equivalent to the succession of "short" trimatrix games, each defined on an interval where the pure strategy value is constant.

Owing to Theorem 5 along with Theorem 3 and Theorem 4 the equilibrium of the initial game can be obtained by stacking the equilibria of the "short" trimatrix games. The stack is always possible, even when only time is discrete (and the set of pure strategy possible values is continuous). Any combination of the respective equilibria of the "short" trimatrix games is an equilibrium of the initial three-person game. Moreover, Theorem 4 allows finding a pure-strategy equilibrium of the initial game by stacking the pure-strategy equilibria of the "short" continuous three-person games.

Solving noncooperative games on a product of staircase-function finite spaces can be similarly studied for the case of any number of players. Then the presented assertions and conclusions should be just adapted to this case. However, Theorems $1-3$, as in the case of trimatrix games, will have tinier practical impact due to the equilibrium singleness is unlikely. Obviously, the equilibrium singleness likelihood expectedly decays as the number of players increases.

\section{Acknowledgments}

This work was technically supported by the Faculty of Mechanical and Electrical Engineering at the Polish Naval Academy, Gdynia, Poland.

\section{References}

[1] Agapova A., Madura J., Market uncertainty and earnings guidance, The Quarterly Review of Economics and Finance, 61, 2016, 97-111.

[2] Edwards R. E., Functional Analysis: Theory and Applications, Holt, Rinehart and Winston, New York City, New York, USA, 1965. 
[3] Gogodze J., Revealed comparative advantage method for solving multicriteria decision-making problems, Foundations of Computing and Decision Sciences, 46(1), 2021, 85-96.

[4] Harsanyi J. C., Selten R., A General Theory of Equilibrium Selection in Games, The MIT Press, Cambridge, MA, 1988.

[5] Hirshleifer D., Jiang D., DiGiovanni Y. M., Mood beta and seasonalities in stock returns, Journal of Financial Economics, 137 (1), 2020, 272-295.

[6] Leinfellner W., Köhler E., Game theory, experience, rationality. Foundations of social sciences, economics and ethics. In honor of John C. Harsanyi, Springer, Netherlands, 1998.

[7] Loesche F., Ionescu T., Mindset and Einstellung Effect, Encyclopedia of Creativity, 3, 2020, 174-178.

[8] Meroni C., Pimienta C., The structure of Nash equilibria in Poisson games, Journal of Economic Theory, 169, 2017, 128-144.

[9] Myerson R. B., Refinements of the Nash equilibrium concept, International Journal of Game Theory, 7 (2), 1978, 73-80.

[10] Nisan N., Roughgarden T., Tardos É., Vazirani V. V., Algorithmic Game Theory, Cambridge University Press, Cambridge, UK, 2007.

[11] Nöldeke G., Peña J., The symmetric equilibria of symmetric voter participation games with complete information, Games and Economic Behavior, 99, 2016, 71-81.

[12] Romanuke V. V., Acyclic-and-asymmetric payoff triplet refinement of pure strategy efficient Nash equilibria in trimatrix games by maximinimin and superoptimality, $K P I$ Science News, 4, 2018, 38-53.

[13] Romanuke V. V., Approximate equilibrium situations with possible concessions in finite noncooperative game by sampling irregularly fundamental simplexes as sets of players' mixed strategies, Journal of Uncertain Systems, 10 (4), 2016, 269-281.

[14] Romanuke V.V., Convergence and estimation of the process of computer implementation of the optimality principle in matrix games with apparent play horizon, Journal of Automation and Information Sciences, 45(10), 2013, 49-56.

[15] Romanuke V. V., Ecological-economic balance in fining environmental pollution subjects by a dyadic 3-person game model, Applied Ecology and Environmental Research, 17 (2), 2019, 1451-1474.

[16] Romanuke V. V., Finite approximation of continuous noncooperative two-person games on a product of linear strategy functional spaces, Journal of Mathematics and Applications, 43, 2020, 123-138.

[17] Romanuke V. V., On the point of generalizing the Walsh functions to surfaces, Herald of Khmelnytskyi national university. Technical sciences, 6(1), 2007, 187-193. 
[18] Romanuke V. V., Theoretic-game methods of identification of models for multistage technical control and run-in under multivariate uncertainties (a Dissertation for the Doctoral Degree of Technical Sciences in Speciality 01.05.02 Mathematical Modeling and Computational Methods), Vinnytsia National Technical University, Vinnytsia, Ukraine, 2014 (in Ukrainian).

[19] Romanuke V. V., Theory of Antagonistic Games, New World - 2000, Lviv, 2010.

[20] Romanuke V. V., Uniform sampling of fundamental simplexes as sets of players' mixed strategies in the finite noncooperative game for finding equilibrium situations with possible concessions, Journal of Automation and Information Sciences, 47(9), 2015, 76-85.

[21] Romanuke V.V., Uniform sampling of the infinite noncooperative game on unit hypercube and reshaping ultimately multidimensional matrices of players' payoff values, Electrical, Control and Communication Engineering, 8, 2015, 13-19.

[22] Vorob'yov N. N., Game theory fundamentals. Noncooperative games, Nauka, Moscow, 1984. (in Russian)

[23] Vorob'yov N. N., Game theory for economists-cyberneticists, Nauka, Moscow, 1985. (in Russian)

[24] Yang J., Chen Y.-S., Sun Y., Yang H.-X., Liu Y., Group formation in the spatial public goods game with continuous strategies, Physica A: Statistical Mechanics and its Applications, 505, 2018, 737-743.

[25] Yanovskaya E. B., Antagonistic games played in function spaces, Lithuanian Mathematical Bulletin, 3, 1967, 547-557.

[26] Young P., Zamir S. (eds.), Handbook of Game Theory. Volume 4, North Holland, 2015.

[27] Zhao R., Neighbour G., Han J., McGuire M., Deutz P., Using game theory to describe strategy selection for environmental risk and carbon emissions reduction in the green supply chain, Journal of Loss Prevention in the Process Industries, 25(6), 2012, 927-936.

[28] Zhou Z., Jin Z., Optimal equilibrium barrier strategies for time-inconsistent dividend problems in discrete time, Insurance: Mathematics and Economics, 94, 2020, $100-108$. 